\title{
STAR FORMATION RATES IN RESOLVED GALAXIES: CALIBRATIONS WITH NEAR- AND FAR-INFRARED DATA FOR NGC 5055 AND NGC 6946*
}

\author{
Yiming Li ${ }^{1}$, Alison F. Crocker ${ }^{1}$, Daniela Calzetti ${ }^{1}$, Christine D. Wilson ${ }^{2}$, Robert C. Kennicutt ${ }^{3}$, Eric J. Murphy ${ }^{4}$, \\ Bernhard R. Brandl ${ }^{5}$, B. T. Draine ${ }^{6}$, M. Galametz ${ }^{3}$, B. D. Johnson ${ }^{7}$, L. Armus ${ }^{8}$, K. D. Gordon ${ }^{9}, 10$, K. Croxall $^{11,12}$,

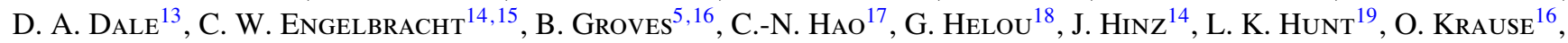 \\ H. Roussel ${ }^{7}$, M. Sauvage ${ }^{20}$, And J. D. T. Smith ${ }^{11}$ \\ ${ }^{1}$ Department of Astronomy, University of Massachusetts, Amherst, MA 01003, USA; yiming1@astro.umass.edu \\ ${ }^{2}$ Department of Physics \& Astronomy, McMaster University, Hamilton, Ontario L8S 4M1, Canada \\ ${ }^{3}$ Institute of Astronomy, University of Cambridge, Madingley Road, Cambridge CB3 OHA, UK \\ ${ }^{4}$ Observatories of the Carnegie Institution for Science, 813 Santa Barbara Street, Pasadena, CA 91101, USA \\ ${ }^{5}$ Leiden Observatory, Leiden University, P.O. Box 9513, 2300 RA Leiden, The Netherlands \\ ${ }^{6}$ Department of Astrophysical Sciences, Princeton University, Princeton, NJ 08544, USA \\ ${ }^{7}$ Institut d'Astrophysique de Paris, UMR7095 CNRS, Université Pierre \& Marie Curie, 98 bis Boulevard Arago, F-75014 Paris, France \\ ${ }^{8}$ Spitzer Science Center, California Institute of Technology, MC 314-6, Pasadena, CA 91125, USA \\ ${ }^{9}$ Space Telescope Science Institute, 3700 San Martin Drive, Baltimore, MD 21218, USA \\ ${ }^{10}$ Sterrenkundig Observatorium, Universiteit Gent, Krijgslaan 281, B-9000 Gent, Belgium \\ ${ }^{11}$ Department of Physics and Astronomy, University of Toledo, Toledo, OH 43606, USA \\ ${ }^{12}$ Department of Astronomy, The Ohio State University, 4051 McPherson Laboratory, Columbus, OH 43210, USA \\ ${ }^{13}$ Department of Physics \& Astronomy, University of Wyoming, Laramie, WY 82071, USA \\ ${ }_{15}^{14}$ Steward Observatory, University of Arizona, Tucson, AZ 85721, USA \\ 15 Raytheon Company, 1151 E. Hermans Road, Tucson, AZ 85756, USA \\ ${ }^{16}$ Max-Planck-Institut für Astronomie, Königstuhl 17, D-69117 Heidelberg, Germany \\ 17 Tianjin Astrophysics Center, Tianjin Normal University, Tianjin 300387, China \\ ${ }^{18}$ NASA Herschel Science Center, IPAC, California Institute of Technology, Pasadena, CA 91125, USA \\ ${ }^{19}$ INAF-Osservatorio Astrofisico di Arcetri, Largo E. Fermi 5, I-50125 Firenze, Italy \\ ${ }^{20}$ CEA/DSM/DAPNIA/Service d'Astrophysique, UMR AIM, CE Saclay, F-91191 Gif sur Yvette Cedex, France \\ Received 2012 November 12; accepted 2013 April 4; published 2013 April 26
}

\begin{abstract}
We use the near-infrared $\mathrm{Br} \gamma$ hydrogen recombination line as a reference star formation rate (SFR) indicator to test the validity and establish the calibration of the Herschel/PACS $70 \mu \mathrm{m}$ emission as a SFR tracer for sub-galactic regions in external galaxies. $\mathrm{Br} \gamma$ offers the double advantage of directly tracing ionizing photons and of being relatively insensitive to the effects of dust attenuation. For our first experiment, we use archival Canada-France-Hawaii Telescope Br $\gamma$ and $K s$ images of two nearby galaxies: NGC 5055 and NGC 6946, which are also part of the Herschel program KINGFISH (Key Insights on Nearby Galaxies: a Far-Infrared Survey with Herschel). We use the extinction corrected $\mathrm{Br} \gamma$ emission to derive the SFR(70) calibration for H II regions in these two galaxies. A comparison of the SFR(70) calibrations at different spatial scales, from 200 pc to the size of the whole galaxy, reveals that about $50 \%$ of the total $70 \mu \mathrm{m}$ emission is due to dust heated by stellar populations that are unrelated to the current star formation. We use a simple model to qualitatively relate the increase of the SFR(70) calibration coefficient with decreasing region size to the star formation timescale. We provide a calibration for an unbiased SFR indicator that combines the observed $\mathrm{H} \alpha$ with the $70 \mu \mathrm{m}$ emission, also for use in $\mathrm{H}$ II regions. We briefly analyze the PACS 100 and $160 \mu \mathrm{m}$ maps and find that longer wavelengths are not as good SFR indicators as $70 \mu \mathrm{m}$, in agreement with previous results. We find that the calibrations show about $50 \%$ difference between the two galaxies, possibly due to effects of inclination.
\end{abstract}

Key words: galaxies: ISM - H II regions - infrared: galaxies - infrared: ISM - ISM: structure

Online-only material: color figures

\section{INTRODUCTION}

Star formation rates (SFRs) have been measured and characterized across all wavelengths, from the X-ray, through the ultraviolet (UV), optical, infrared (IR), and all the way to the radio (for a review, see Kennicutt \& Evans 2012 for the past decade, and Kennicutt 1998 for earlier literature). Thanks to the synergy between Spitzer, GALEX, and Herschel, many SFR indicators have been recently defined and/or re-calibrated at UV, optical,

\footnotetext{
* Based on observations obtained with WIRCam, a joint project of CFHT, Taiwan, Korea, Canada, France, and the Canada-France-Hawaii Telescope (CFHT) which is operated by the National Research Council (NRC) of Canada, the Institute National des Sciences de l'Univers of the Centre National de la Recherche Scientifique of France, and the University of Hawaii.
}

and IR wavelengths (e.g., Wu et al. 2005; Alonso-Herrero et al. 2006; Calzetti et al. 2007, 2010; Rieke et al. 2009; Kennicutt et al. 2007, 2009; Boquien et al. 2010; Lawton et al. 2010; Li et al. 2010; Hao et al. 2011; Murphy et al. 2011b). However, any new calibration or re-calibration needs a "reference" SFR indicator against which it can be compared. Different authors use diverse reference SFR indicators (extinction-corrected $\mathrm{H} \alpha$, total IR, radio, combination of $\mathrm{H} \alpha$ and $24 \mu \mathrm{m}$ emission, etc.); their choices are usually driven by the available data sets. Thus, newly (re)calibrated SFR tracers are not usually comparable, to each other or to more established indicators. Furthermore, their accuracy and range of applicability often cannot be reliably established. For example, to calibrate an IR SFR indicator with a reference SFR indicator involving another IR band (e.g., 
the combination of $\mathrm{H} \alpha$ and $24 \mu \mathrm{m})$, degeneracy may arise at high SFR regime where the dust emission dominates. A lack of a robust, wide field, reference SFR renders the absolute calibration and universality of the $24 \mu \mathrm{m}$ term in hybrid tracers like $\mathrm{H} \alpha+24 \mu \mathrm{m}$ and FUV+24 $\mu \mathrm{m}$ uncertain, even for the wellstudied SINGS sample (Leroy et al. 2012). Progress in calibrating SFR indicators at a variety of wavelengths requires the use of "unbiased" reference SFR tracers, i.e., those insensitive to dust extinction, star formation history, etc.

Near-infrared (NIR) hydrogen recombination lines, such as $\mathrm{P} \alpha(1.8756 \mu \mathrm{m}$, from the Hubble Space Telescope (HST)) and $\operatorname{Br} \gamma(2.166 \mu \mathrm{m})$, and the radio free-free emission (e.g., with the Green Bank Telescope (GBT); Murphy et al. 2011b, 2012), trace the ionizing photons produced by massive stars and therefore the most recent ( $<10 \mathrm{Myr}$ ) star formation in a galaxy. As such, these tracers are less affected by variations in the star formation history of a region or galaxy or, as in the case of IR SFR tracers, by variations in the dust content (Kennicutt \& Evans 2012; Calzetti 2012). They are also significantly less affected by dust extinction than $\mathrm{UV}$ and $\mathrm{H} \alpha$ emission; e.g., dust extinction is significantly lower at $\mathrm{Br} \gamma: A_{\mathrm{H} \alpha}=1 \mathrm{mag}$ corresponds to $A_{\mathrm{Br} \gamma}=$ 0.14 mag. The NIR hydrogen recombination lines are also free of in-band contamination from adjacent emission lines (e.g., [N II] for $\mathrm{H} \alpha$ or non-thermal emission for the radio free-free emission). $\mathrm{P} \alpha$ and radio free-free emission have both previously been used as a reference SFR to calibrate or study other SFR indicators (Calzetti et al. 2007; Murphy et al. 2011b). However, the use of these two indicators is limited due to the small field of view (FOV) of the P $\alpha$ observations with HST (e.g., Calzetti et al. 2007 only studied the central 1-2 kpc of star-forming galaxies), and the time-consuming nature of free-free observation to map whole galaxies.

The WIRCam (Wide Infrared Camera) on CFHT (CanadaFrance-Hawaii Telescope) provides the capability to observe the NIR hydrogen recombination line, Brackett- $\gamma(\mathrm{Br} \gamma, 2.166 \mu \mathrm{m}$; Jones et al. 2002), from the ground with large enough FOV $\left(20^{\prime} \times 20^{\prime}\right)$ to cover most nearby galaxies out to $R_{25}$ with one pointing $\left(R_{25}\right.$ is defined as the $25 \mathrm{mag} \operatorname{arcsec}^{-2}$ isophote). Much like $\mathrm{P} \alpha, \mathrm{Br} \gamma$ emission traces the ionizing photons from massive stars; it has the advantage of mapping efficiency over $H S T \mathrm{P} \alpha$ or $\mathrm{P} \beta$ (Wide Field Camera 3, WFC3) observations thanks to the large FOV of modern IR detectors. The lower angular resolution provided by ground-based observations relative to the HST is, however, more than sufficient for our planned calibration of far-infrared (FIR) emission.

High-sensitivity, high-angular resolution IR telescopes, like the Spitzer and, more recently, the Herschel space telescopes, have provided a major incentive to investigate dust-obscured star formation in galaxies at all cosmic times, and at all scales, from galaxy-integrated to sub-galactic scales. Both telescopes have shown that dust-obscured starbursts dominate the star formation in the redshift range 1-3 (Le Floc'h et al. 2005; Magnelli et al. 2009; Elbaz et al. 2011; Murphy et al. 2011a; Reddy et al. 2012). Concurrently, studies of nearby galaxies at a few hundred parsec (pc) resolution with Spitzer and GALEX have yielded new insights on the fundamental processes responsible for converting gas into stars and the Schmidt Law. Data finally probe the physical scales of Giant Molecular Associations ( 100-200 pc; Koda et al. 2009), where models of star formation can be discriminated (Kennicutt et al. 2007; Leroy et al. 2008; Liu et al. 2011; Rahman et al. 2012; Calzetti et al. 2012). Calibrations of SFR indicators in the FIR ( $\gtrsim 40-50 \mu \mathrm{m})$ regime at sub-galactic scales have thus become useful for these kinds of studies, leading
Table 1

Multi-wavelength Data

\begin{tabular}{lccc}
\hline \hline $\begin{array}{l}\text { Data } \\
(1)\end{array}$ & Instrument & Central Wavelength & PSF \\
\hline $\mathrm{Br} \gamma$ & $(2)$ & $(3)$ & $(4)$ \\
$K s$ & CFHT/WIRCam & $2.166 \mu \mathrm{m}$ & $\sim 1^{\prime \prime} .1$ \\
$\mathrm{H} \alpha$ & CFHT/WIRCam & $2.146 \mu \mathrm{m}$ & $\sim 1^{\prime \prime}$ \\
$70 \mu \mathrm{m}$ & KPNO 2.1 m/2.3 m Bok & $6573 \AA$ & $\sim 1^{\prime \prime}$ \\
$100 \mu \mathrm{m}$ & Herschel/PACS & $70 \mu \mathrm{m}$ & $5^{\prime \prime} .5$ \\
$160 \mu \mathrm{m}$ & Herschel/PACS & $100 \mu \mathrm{m}$ & $6^{\prime \prime} .9$ \\
Free-free emission & Herschel/PACS & $160 \mu \mathrm{m}$ & $11^{\prime \prime} 5$ \\
& GBT Ka-band & $33 \mathrm{GHz}$ & $25^{\prime \prime}$ \\
\hline
\end{tabular}

Notes. Column 1: data name; Column 2: telescope, instrument, and filter; Column 3: filter's central wavelength; Column 4: FWHM of the PSF.

recent studies to focus on them (Calzetti et al. 2010; Lawton et al. 2010; Li et al. 2010). The integrated FIR emission from whole galaxies contains significant diffuse emission that comes from dust heated by stellar populations not related to the current star formation. A comparison in Li et al. (2010) between the calibrations of SFR(70) for both sub-galactic regions and entire galaxies reveals that, on kiloparsec scales and larger, about $40 \%$ of emission of $70 \mu \mathrm{m}$ in the galaxies comes from diffuse emission, on average.

In this paper, we use $\mathrm{Br} \gamma$ maps of two KINGFISH (Key Insights on Nearby Galaxies: a Far-Infrared Survey with Herschel; Kennicutt et al. 2011) galaxies, retrieved from the CFHT archive: NGC 5055 and NGC 6946, to calibrate the Herschel/PACS $70 \mu \mathrm{m}$ band as a SFR indicator at sub-galactic scales. We also consider the 100 and $160 \mu \mathrm{m}$ Herschel bands as SFR indicators. The resolution of Herschel at $70 \mu \mathrm{m}$ (FWHM $\left.5^{\prime \prime} .5\right)$ enables us to resolve regions as small as $200 \mathrm{pc}$ in these two galaxies. This scale is considerably smaller than a galaxy, and although several $\mathrm{H}$ II regions will be included, we expect the physical characteristics of the stellar population in these apertures to be different from those of the entire galaxy. This paper is devoted to quantifying the differences of the calibrations between the whole galaxies and sub-galactic regions, and to providing interpretation with model comparisons.

Units in this paper are as follows: $\mathrm{erg} \mathrm{s}^{-1}$ for luminosity, erg s${ }^{-1} \mathrm{kpc}^{-2}$ for luminosity surface density (LSD, with symbol $\Sigma), M_{\odot} \mathrm{yr}^{-1}$ for SFR and $M_{\odot} \mathrm{yr}^{-1} \mathrm{kpc}^{-2}$ for SFR surface density ( $\Sigma($ SFR) or SFRD), unless otherwise specified. The IR luminosity used in the paper of the IR bands is, $L(\mathrm{IR})=v L_{v}$, following the common definition of monochromatic IR flux.

\section{DATA}

Table 1 contains a summary of the data involved in the analyses in this paper, along with their angular resolutions. Detailed descriptions are given in following sections.

\subsection{Archive CFHT Data}

Two galaxies, NGC 5055 and NGC 6946 (Table 2), out of the KINGFISH sample of 61 galaxies have archival observations of sufficient depth $(>1000 \mathrm{~s}$ integration time in the $\mathrm{Br} \gamma$ narrowband, see Section 2.1.3 for the final noise levels of the reduced images) for our purposes with WIRCam (Puget et al. 2004), observed by PI Daniel Devost with RUNID 07AD86 for NGC 5055 and 07BD91 for NGC 6946. WIRCam is the NIR wide-field imager on the CFHT and has been in operation since 2005 November. It contains four $2048 \times 2048$ pixel 


\begin{tabular}{|c|c|c|c|c|c|c|c|c|c|}
\hline \multirow{3}{*}{$\begin{array}{l}\text { Galaxy } \\
\text { (1) }\end{array}$} & \multirow{3}{*}{$\begin{array}{l}\text { Type } \\
\text { (2) }\end{array}$} & \multirow{3}{*}{$\begin{array}{c}\text { Distance } \\
(\mathrm{Mpc}) \\
(3)\end{array}$} & \multirow{3}{*}{$\begin{array}{c}\text { Diameter } \\
\left({ }^{\prime} x^{\prime}\right) \\
(4)\end{array}$} & \multicolumn{3}{|c|}{$\begin{array}{l}\text { Table } 2 \\
\text { Sample }\end{array}$} & \multirow{3}{*}{$\begin{array}{c}{[\mathrm{N} \text { II }] / \mathrm{H} \alpha} \\
(8)\end{array}$} & \multirow{2}{*}{\multicolumn{2}{|c|}{$E(B-V)$}} \\
\hline & & & & $i$ & $12+1$ & $\mathrm{O} / \mathrm{H})$ & & & \\
\hline & & & & $\begin{array}{l}(\mathrm{deg}) \\
(5)\end{array}$ & $\begin{array}{l}(\mathrm{PT}) \\
(6)\end{array}$ & $\begin{array}{c}(\mathrm{KK}) \\
(7)\end{array}$ & & $\begin{array}{c}\text { Foreground } \\
\text { (9) }\end{array}$ & $\begin{array}{c}\text { Regions } \\
\text { (10) }\end{array}$ \\
\hline NGC 5055 & SAbc & 7.94 & $12.6 \times 7.2$ & 56 & 8.40 & 9.14 & $0.486 \pm 0.019$ & 0.018 & 0.65 \\
\hline NGC 6946 & SABcd & 6.8 & $11.5 \times 9.8$ & 32 & 8.40 & 9.05 & $0.448 \pm 0.087$ & 0.303 & 0.44 \\
\hline
\end{tabular}

Notes. Column 1: galaxy name; Column 2: galaxy type; Column 3: galaxy distance from Kennicutt et al. (2011); Column 4: galaxy sizes from Kennicutt et al. (2011); Column 5: inclination from Moustakas et al. (2010); Columns 6 and 7: characteristic oxygen abundances of the galaxies; the two columns, (PT) and (KK), are the two oxygen abundances listed in Table 9 of Moustakas et al. (2010): the PT value, in the left-hand-side column, is from the empirical calibration of Pilyugin \& Thuan (2005); the KK value, in the right-hand-side column, is from the theoretical calibration of Kobulnicky \& Kewley (2004); Column 8: [N II]/H $\alpha$ ratio from Kennicutt et al. (2009); Column 9: $E(B-V)$ used for foreground Galactic extinction correction from NED (Schlegel et al. 1998; Schlafly \& Finkbeiner 2011); Column 10: median $E(B-V)$ derived from $\mathrm{H} \alpha$ and $\mathrm{Br} \gamma$ luminosity for selected $\mathrm{H}$ II regions at $70 \mu$ m resolution, after removal of the Galactic foreground extinction (Schlegel et al. 1998; O’Donnell 1994).

Table 3

Archival CFHT Images

\begin{tabular}{|c|c|c|c|c|c|}
\hline \multirow[b]{2}{*}{$\begin{array}{l}\text { Galaxy } \\
\text { (1) }\end{array}$} & \multicolumn{2}{|r|}{$K s$} & \multicolumn{2}{|r|}{$\mathrm{Br} \gamma$} & \multirow{2}{*}{$\begin{array}{l}\text { Note } \\
\text { (6) }\end{array}$} \\
\hline & $\begin{array}{l}\# \\
(2)\end{array}$ & $\begin{array}{c}\text { Exposure Time (s) } \\
\text { (3) }\end{array}$ & $\begin{array}{c}\# \\
(4)\end{array}$ & $\begin{array}{c}\text { Exposure Time (s) } \\
(5)\end{array}$ & \\
\hline NGC 5055 & 112 & 20 & $99^{\mathrm{a}}$ & 100 & RUNID:07AD86 \\
\hline NGC 6946 & 61 & 10 & 61 & 110 & RUNID:07BD91 \\
\hline
\end{tabular}

Notes. Column 1: galaxy name; Columns 2 and 3: number of images and exposure time with the $K s$ broadband filter; Columns 4 and 5: number of images and exposure time with the $\mathrm{Br} \gamma$ narrowband filter; Column 6: observation information.

a Twelve of the narrowband exposures for NGC 5055 have exposure times of $200 \mathrm{~s}$, and we rescale them to $100 \mathrm{~s}$ exposure maps.

HAWAII2-RG detectors and covers a total $20^{\prime} \times 20^{\prime}$ FOV with a sampling of 0.3 arcsec per pixel (see Table 2 for the angular sizes of the two galaxies).

The observations of both galaxies were performed by sequentially shifting the object into the center of each detector for each new exposure. Additionally, a certain amount of dithering around the object was added to mitigate the effect of bad pixels. This chip-shifting method allows adjacent off-target exposures of the same detector to be used for sky subtraction as the two galaxies both have angular sizes comparable to the size of one detector. Pipeline processed images with sky subtraction (including dark subtraction, flat-fielding with dome flat exposures, basic crosstalk removal, Two Micron All Sky Survey absorption estimate and sky subtraction using neighboring images) are retrieved from the CFHT science data archive and the total number of exposures is listed in Table 3. The actual pixel scale of the retrieved images is 0.304 arcsec per pixel. Each exposure is stored in a multi-extension fits file with four frames corresponding to the four different chips. As each exposure contains only one on-target frame, with the off-target frames used for background subtraction, we have a total of 99 narrowband and 112 broadband sky subtracted frames for NGC 5055 and 61 narrowband and 61 broadband sky subtracted frames for NGC 6946.

In this paper we use "narrowband map" to refer to the observed line-plus-continuum frames obtained with the $\mathrm{Br} \gamma$ filter (central wavelength $2166 \mathrm{~nm}$, bandwidth $29.5 \mathrm{~nm}$ ), " $\mathrm{Br} \gamma$ map" to refer to the $\mathrm{Br} \gamma$ emission line-only frames, and "broadband map" to refer to the observed continuum frames obtained with the $K s$ filter.

\subsubsection{NGC 5055 Reduction}

For the observations of NGC 5055, the different exposures from the same detector are well aligned (as reduced by the CFHT pipeline), but not for exposures from different detectors. So we first combine all the images from the same detector into one single image using IRAF. A sigma clipping algorithm is used to eliminate spurious features, mostly the cross-talk from bright stars. All the sky subtracted CFHT images are given a "chipbias" to prevent 16 bit wrapping of negative numbers into high positive numbers and all the bad pixels are given a value of 0 , so a global "sky" value for each image, the mode ${ }^{21}$ value of the whole map, with values close to the "chipbias," is first subtracted from each image. This process reduces our collection of NGC 5055 images from 99/112 (narrowband/broadband) to eight: one combined frame for each detector and each filter. The relative astrometry between the two bands of the same detector is stable so we use the broadband images to derive the alignment transformation between detectors and apply them to both the broadband and narrowband images. Thirty point-like sources (foreground stars) are chosen near and within the galaxy and the coordinates of their centers are determined. These coordinates are then used as input to the IRAF tasks "geomap" and "geotran" to align three of the images relative to the fourth one, with a manual inspection of the geometrical transformation process to mask out outliers due to mis-centering. A higher order fit is used to allow both offsets and rotation/stretching to be corrected. The same transformation is applied to align three of the narrowband images, relative to the fourth one. The four images from the four different detectors are then combined for the narrowband and the broadband separately.

The chip1 and chip4 Ks images of NGC 5055 suffer from severe crosstalk from the bright guide star and thus only the chip 2 and chip $3 K s$ images are used for this galaxy. This reduced the number of images combined to produce the $K s$ image to fiftysix 20 s exposures. However, for the sole purpose of continuum subtraction of the $\mathrm{Br} \gamma$ image, the resultant image still provides sufficient depth (see Section 2.1.3).

\subsubsection{NGC 6946 Reduction}

For this galaxy we had to proceed with a different approach than for NGC 5055 as the different exposures, even from the same detector, is not well aligned. No reason is noted on the

\footnotetext{
21 Mode is the global peak of the value distribution. It is robust against the tail of positive sources to estimate the sky value.
} 
archival images but possibly it is because of the large dither offset from one exposure to the other. Thus, for the NGC 6946 images, we manually identified 137 point-like sources across the whole field, in order to align all images relative to each other and across the entire FOV, also correcting for distortions. The IRAF tasks "geomap" and "geotran" are again used, with manual inspection, to align the 61 images with reference to the first image for both the narrowband and the broadband separately. The rest of the approach remains the same as that of NGC 5055 and in this case there is no problem with crosstalk from the guide star. Then the 61 images are combined using IRAF task "imcombine" for the two bands separately.

\subsubsection{Continuum Subtraction and Photometric Calibration}

To obtain the $\mathrm{Br} \gamma$ emission line image, we remove the underlying stellar continuum from the narrowband image. We use the broadband $K s$ image for this purpose. Both the narrowband image and the broadband image are first rescaled by the exposure time, and the $K s$ image resolution is matched to the $\mathrm{Br} \gamma$ image resolution with the IRAF task "psfmatch," with a set of point-like sources $(\sim 10$, these are presumably late-type main sequence stars in our own Galaxy, and are not saturated in our images). The stars have FWHMs from 1". 1 to 1 1.4, and the differences between the two bands are less than 0.2 . Then, photometry of the same set of stars for both NGC 5055 and NGC 6946, respectively, is performed in both bands; the average narrowband-to-broadband fractions are determined for both galaxies separately. The average fractions serve as a fiducial number we use to perform the continuum subtraction, assuming the $\mathrm{Br} \gamma$ features from the stars are negligible.

Many factors may actually affect the accuracy of this number, such as spectral type differences between stars and $\mathrm{H}$ II regions, differential foreground extinction, etc. The $K s$ band targets mostly the Rayleigh-Jeans tail of stellar emission, and, for our galaxies, we do not expect large in-band color variations due to variations in stellar populations or due to the presence of spatially variable dust attenuation. Stellar population synthesis models (Starburst99) indicate that variations in the stellar flux ratio between the two wavelength extremes of the $K s$ filter are less than 3\% for an age range between 6 Myr and $10 \mathrm{Gyr}$. We verify the impact of in-band dust attenuation variations by considering our largest $A_{V}=3.5 \mathrm{mag}$ derived from $\mathrm{H} \alpha / \mathrm{Br} \gamma$. This corresponds to $A_{K s} \sim 0.18 \mathrm{mag}$, when including the factor $\sim 2$ reduction in attenuation for the stellar continuum relative to the ionized gas (Calzetti et al. 2000; Hao et al. 2011). The variations in stellar color across the $K s$ filter due to this value of $A_{K s}$ is about $1 \%$. We also cross-check these scaling numbers against an optical continuum subtraction method (S. Hong et al., in preparation), which analyzes the statistics of the continuum subtracted images with different continuum fractions and gives a robust estimate of the acceptable continuum fraction range. The acceptable range we get from this method includes our estimate from rescaling star fluxes, which shows that the two methods agree. Having performed this consistency check, we ultimately use the factor determined from the stars and inspect the continuum subtracted $\mathrm{Br} \gamma$ images to make sure the subtraction looks reasonable. The fractions of the broadband fluxes adopted are 0.0890 and 0.0864 for NGC 5055 and NGC 6946, respectively. The contribution of $\mathrm{Br} \gamma$ lines to the $K s$ filter (FWHM $315 \mathrm{~nm}$ ) is negligible ( $\leqslant 1.5 \%$ even for the youngest star forming regions), thus it is not necessary to correct for the over-subtraction of the $\mathrm{Br} \gamma$ line itself when subtracting the continuum. In addition to the $\mathrm{Br} \gamma$ line, the $\mathrm{H}_{2}$ rotational transitions may contribute to the emission in the $K s$ filter. These, however, tend to contribute at most $2.4 \%$, and more typically less than $1 \%$, to the total $K s$ band, even in active starburst systems (Calzetti 1997; Rosenberg et al. 2013).

Despite the care taken in the continuum subtraction, we still test for the potential effect of incorrect continuum subtraction. For larger the determined value of continuum subtraction fraction, we find the smallest multiplicative value that gives over-subtracted $\mathrm{Br} \gamma$ images for the two galaxies; this value is taken as the upper bound of possible continuum subtraction fractions. The symmetric value on the smaller side relative to this is taken as the lower bound, as it is more difficult to determine the point where under-subtraction occurs. The adopted ranges are 0.0880 to 0.0900 and 0.0852 to 0.0876 for NGC 5055 and NGC 6946, respectively. A set of aperture photometry measurements on $\mathrm{H}$ II regions are performed on images with different continuum subtraction levels (the optimal value and the two boundaries determined above), which reveals an average of $10 \%-15 \%$ variation in flux measurement between the optimal subtraction fraction and the boundaries. This variation depends on how bright the sources are and the difference could rise to nearly a factor of two for the extremely faint HiI regions, with low signal-to-noise ratio $(\mathrm{S} / \mathrm{N} \lesssim 1)$. We thus adopt a $\mathrm{S} / \mathrm{N}>3$ cut that immediately excludes such extreme faint regions (see Section 3.3.2). The central parts of both galaxies and the centers of the bright stars show artifacts on the lineonly $\mathrm{Br} \gamma$ maps, mainly due to saturation of these regions. The artifacts may also be enhanced by small misalignments in the final combined images, because the centroids are poorly determined in saturated sources. We make no use of the bright stars and try to avoid the regions (central $\sim 10^{\prime \prime}$ ) affected by these artifacts in our analysis. Also, because NGC 6946 is in a very crowded field, the foreground stars with obvious residual after continuum subtraction are masked out. The final $\mathrm{Br} \gamma$ images for NGC 5055 and NGC 6946 both show an uneven background, possibly due to an imperfect sky subtraction in the pipeline and the interpolation in the process of aligning the different exposures. To flatten the background, we use the IRAF package "imsurfit" to fit a two-dimensional low-order surface and subtract the background from the images. Although this approach is effective at flattening the line-only images, it may not be sufficient for large scale analysis (the peak-to-peak difference in the background for the images matched to the resolution of the IR data is about $5 \sigma$ ). We thus avoid deriving integrated luminosities for the whole galaxy or radial trends, and limit our analysis to the typical (or somewhat larger) sizes of $\mathrm{H}$ II regions.

The $\mathrm{Br} \gamma$ maps are calibrated with the header keyword "phot_c0," which is determined using four well modeled spectrophotometric standards (CALSPEC models) and filter transmission curves, with the information from the WIRCam site $^{22,23,24}$. A comparison between this calibration and the $H S T$ $\mathrm{P} \alpha$ fluxes for these two galaxies, assuming Case-B (Osterbrock 1989) recombination conditions and similar extinction at $\mathrm{P} \alpha$ $(1.8756 \mu \mathrm{m})$ and $\operatorname{Br} \gamma(2.166 \mu \mathrm{m})$, shows consistency within $5 \%$. The uncertainty of "phot_c0" is around 0.1 magnitude $(\sim 10 \%)$. Including the uncertainty from continuum subtraction, we expect about a $15 \%$ uncertainty in the absolute flux

\footnotetext{
22 http://www.cfht.hawaii.edu/Instruments/Imaging/WIRCam/ WIRCamStandardstars.html

23 http://www.cfht.hawaii.edu/Instruments/Imaging/WIRCam/ dietWIRCam.html\#UI

${ }^{24}$ http://www.cfht.hawaii.edu/Instruments/Filters/wircam.html
} 
calibration (sum in quadrature of $10 \%$ from the calibration keyword uncertainty and about $10 \%$ from the continuum subtraction uncertainty), which could be less for bright $\mathrm{H}$ in regions (close to $\sim 10 \%$ ) as the continuum subtraction mainly affects the fainter regions. The final continuum subtracted images have $1 \sigma$ noise level of $2.12 \times 10^{-18} \mathrm{erg} \mathrm{s}^{-1} \mathrm{~cm}^{-2}$ pixel $^{-1}(0.3$ arcsec per pixel $)$ for NGC 5055 and $2.48 \times 10^{-18} \mathrm{erg} \mathrm{s}^{-1} \mathrm{~cm}^{-2}$ pixel $^{-1}$ for NGC 6946.

\subsection{Herschel/PACS Data}

Herschel/PACS observations in all three bands (70, 100, and $160 \mu \mathrm{m})$ are available for both galaxies from the KINGFISH project. The observational program and data processing procedures for KINGFISH are described in detail in Kennicutt et al. (2011); Dale et al. (2012). Briefly, PACS imaging was obtained with $15^{\prime}$ long scan maps, along two perpendicular axes for improved image reconstruction, at the medium scan speed of $20^{\prime \prime} \mathrm{s}^{-1}$. The $45^{\circ}$ orientation of the array with respect to the scan direction contributes to a more uniform spatial coverage. The raw ("Level 0") data were processed using version 5 of HIPE (Ott 2010). Besides the standard pipeline procedures, the conversion from Level 0 to Level 1 data included second level deglitching and corrections for any offsets in the detector submatrices. Scanamorphos ${ }^{25}$ (Roussel 2012) was used to process the Level 1 PACS scan map data to Level 2 final products, where the pixel size is $\sim$ one-fourth of the beam FWHM, i.e., 1".4 at $70 \mu \mathrm{m}, 1^{\prime \prime} .7$ at $100 \mu \mathrm{m}$, and 2". 85 at $160 \mu \mathrm{m}$. A multiplicative factor was also implemented to update the PACS calibration, which is equivalent to the version 26 calibration. ${ }^{26}$ We adopt the latest values of $10 \%$ for all three PACS bands as absolute calibration uncertainty for our extended sources ${ }^{27,28}$. The data used in this paper are processed with Scanamorphos version 12.5 while the most recent KINGFISH data are processed with Scanamorphos version 16.9; checks performed against the versions 16.9 of the Herschel maps show $<1 \%$ difference in photometry.

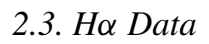

To correct for the small amount of dust extinction in our $\mathrm{Br} \gamma$ maps, we use the H $\alpha$ images of NGC 6946 from the Spitzer Infrared Nearby Galaxies Survey (SINGS; Kennicutt et al. 2003) and of NGC 5055 from the Local Volume Legacy (LVL; Kennicutt et al. 2008; Dale et al. 2009; Lee et al. 2011) survey (Figures 1 and 2). The $\mathrm{H} \alpha$ photometry is corrected for [N II] contamination using the $[\mathrm{N} \mathrm{II}] / \mathrm{H} \alpha$ ratios listed in Kennicutt et al. (2009, and references therein; and also in Table 2). The SINGS $\mathrm{H} \alpha$ map of NGC 6946 only covers the central $\sim 15 \mathrm{kpc}$ by $15 \mathrm{kpc}$ (about $450^{\prime \prime}$ by $450^{\prime \prime}$ ) area of the galaxy (Figure 1), and we limit our analysis to this region. We use the $\mathrm{H} \alpha$ map to correct for dust attenuation and the typical attenuation corrections of $\mathrm{H}$ II regions are $A(\mathrm{H} \alpha) \sim 1.65 \mathrm{mag}$ for NGC 5055 and $A(\mathrm{H} \alpha) \sim$ $1.12 \mathrm{mag}$ for NGC 6946 (see Section 2.3.1 and Table 2), after removal of foreground Galactic extinction, as derived using the method described in the following section.

\footnotetext{
$25 \mathrm{http} / / / \mathrm{www} 2$. iap.fr/users/roussel/herschel

$26 \mathrm{http} / / /$ herschel.esac.esa.int/twiki/bin/view/Public/

PacsCalTreeHistory?template $=$ viewprint

27 https://nhscsci.ipac.caltech.edu/pacs/docs/Photometer/ PICC-NHSC-TR-034.pdf

${ }^{28}$ http://herschel.esac.esa.int/twiki/pub/Public/PacsCalibrationWeb/ ExtSrcPhotom.pdf
}

\subsubsection{Extinction Correction}

With a simple Case $\mathrm{B}$ recombination assumption, the ratio of $\mathrm{H} \alpha$ emission to $\mathrm{Br} \gamma$ emission from the same $\mathrm{H}$ II region is expected to be $\sim 103$ (Osterbrock 1989) for an H II region with electron temperature of $10,000 \mathrm{~K}$ and electron density of $10^{2} \mathrm{~cm}^{-3}$, changing by only $35 \%$ for electron temperatures in the range $5000-20,000 \mathrm{~K}$ and by only $2 \%-3 \%$ for electron densities in the range $10^{2}-10^{4} \mathrm{~cm}^{-3}$. Figure 3 shows the ratio of $\Sigma(\mathrm{H} \alpha$, obs $)$ to $\Sigma(\mathrm{Br} \gamma$, obs $)$ as a function of $\Sigma(70)$, where the data should lie about the $y=103$ line if there were no extinction. In general, the data for both galaxies show a weak trend for decreasing ratio, thus increasing extinction, for increasing $\Sigma(70)$. The ratio is mostly below 103, showing that extinction is present. Although a couple of regions in NGC 6946 have a ratio greater than 103 , they all have significantly larger error bars indicating lower $\mathrm{S} / \mathrm{N}$ in at least one of the two line measurements.

We use the deviation of the line ratio from a value of 103 to estimate the extinction of both bands. Since $A(\mathrm{H} \alpha)=$ $2.54 \times E(B-V)$ and $A(\mathrm{Br} \gamma)=0.36 \times E(B-V)$, then we can get the nebular gas color excess as

$$
E(B-V)=\frac{\log \left(103 \frac{\Sigma(\mathrm{Br} \gamma, \mathrm{obs})}{\Sigma(\mathrm{H} \alpha, \mathrm{obs})}\right)}{0.87}
$$

where $\Sigma(\mathrm{Br} \gamma, \mathrm{obs}) / \Sigma(\mathrm{H} \alpha$, obs $)$ is the observed surface luminosity ratio. We calculate the color excess for each region and apply the extinction correction to $\Sigma(\mathrm{Br} \gamma)$. The color excess is calculated under the assumption of foreground dust extinction (Calzetti et al. 2000) because the dust extinction corrections in the NIR regime are relatively insensitive to the dust geometry (Calzetti et al. 1996). The extinction corrected $\Sigma(\mathrm{Br} \gamma)$ is then used as a reference SFR indicator. The median $E(B-V)$ values for our regions of NGC 5055 and NGC 6946 at $70 \mu \mathrm{m}$ resolution are listed in Table 2, and correspond to a median of $23.6 \%$ and $15.7 \%$ correction of the $\mathrm{Br} \gamma$ luminosity for NGC 5055 and NGC 6946, respectively.

$\mathrm{H}$ II regions in metal-rich galaxies like NGC 5055 and NGC 6946 often have measured electron temperatures that are slightly lower than our default $10,000 \mathrm{~K}$. A temperature of $8000 \mathrm{~K}$ is more common (Bresolin et al. 2004), which yields a $\mathrm{H} \alpha$ to $\mathrm{Br} \gamma$ ratio of 99.3 . For this case, the derived $E(B-V)$ from Equation (1) would be about 5\% lower and the extinction correction about $1 \%$ lower for the $\mathrm{Br} \gamma$. Given that the conversion from $\mathrm{Br} \gamma$ luminosity to $\mathrm{SFR}(\mathrm{Br} \gamma)$ through $\mathrm{H} \alpha$ luminosity would also be about $4 \%$ lower $(99.3 / 103=0.964)$, the $\operatorname{SFR}(\operatorname{Br} \gamma)$ will be about $5 \%$ lower and thus all the derived parameters which are proportional to $\operatorname{SFR}(\mathrm{Br} \gamma)$ in the rest of the paper would be about $5 \%$ lower. Since this does not have a major impact, we keep our default $\mathrm{H} \alpha$ to $\mathrm{Br} \gamma$ ratio of 103 corresponding to an electron temperature of $10,000 \mathrm{~K}$, for generality.

\section{ANALYSIS}

\subsection{Robustness of the Bry Data}

In order to evaluate our $\mathrm{Br} \gamma$ data reduction and calibration, we derive luminosity functions for the H II regions in the two galaxies (Section 3.1.1). We also compare a small subset of bright H II regions in NGC 6946 with published radio free-free measurements (Section 3.1.2). 


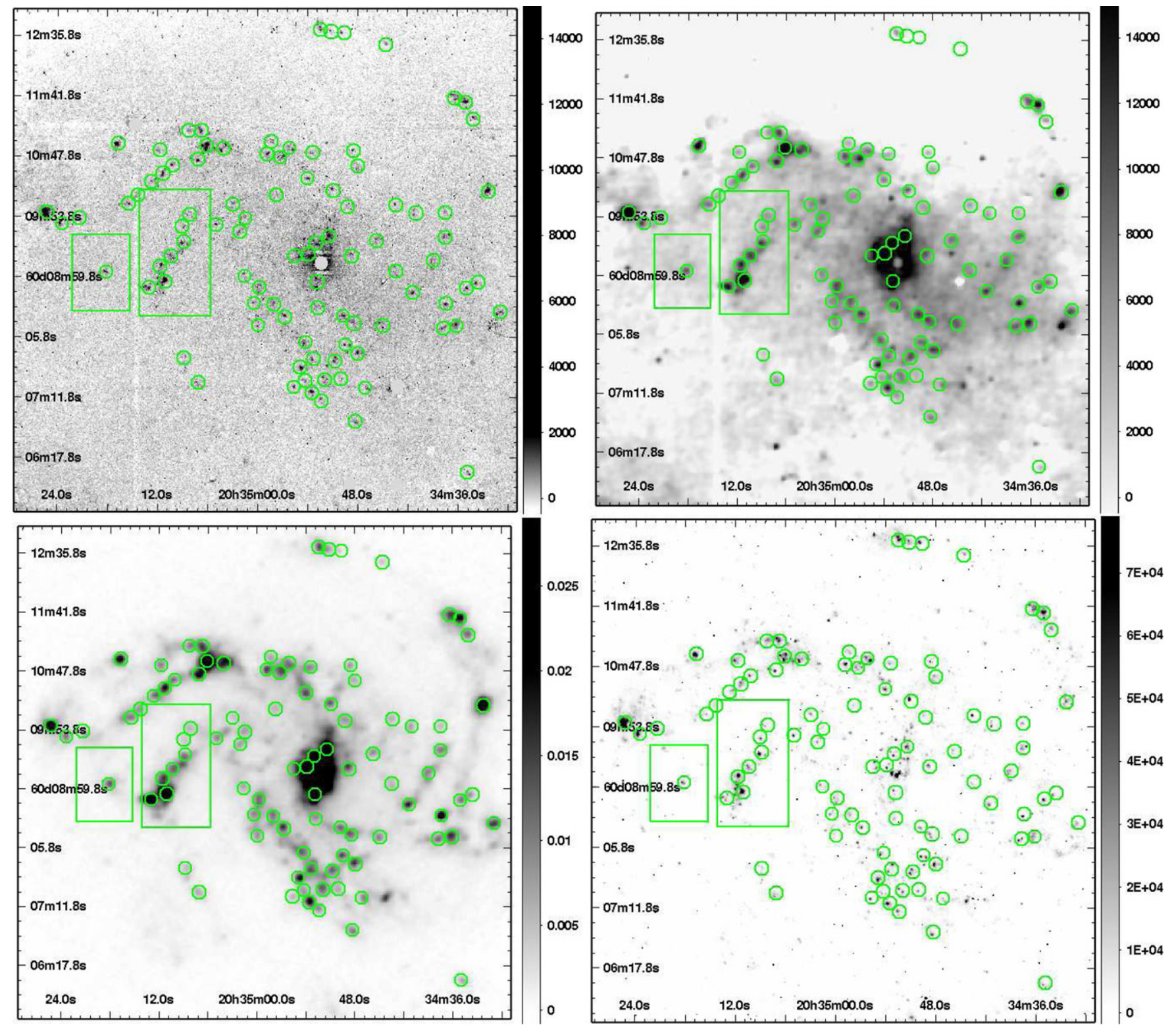

Figure 1. From top left to bottom right: $\mathrm{Br} \gamma, \mathrm{Br} \gamma$ at $70 \mu \mathrm{m}$ resolution, $70 \mu \mathrm{m}$ and $\mathrm{H} \alpha$ images for NGC 6946. The green circles are the apertures we adopted for our analysis at $70 \mu \mathrm{m}$ resolution. The boxes are the local regions, with relatively even background, selected for the comparison between the two methods of subtracting the local background (Section 3.3.1). North is up and east is left. The image size is about $450^{\prime \prime} \times 450^{\prime \prime}$ (about $14.8 \times 14.8 \mathrm{kpc}$ ) for each panel. The uneven background of the $\mathrm{Br} \gamma$ images is greatly enhanced after convolving to the $70 \mu \mathrm{m}$ resolution. The units for the grayscale bars are, $10^{-20} \mathrm{erg} \mathrm{s}^{-1} \mathrm{~cm}^{-2}$ per pixel for Br $\gamma$ and $\mathrm{H} \alpha$ with pixel sizes of 0!'304 and for $\mathrm{Br} \gamma$ at $70 \mu \mathrm{m}$ resolution with a pixel size of 1".4, and Jansky (Jy) per pixel for $70 \mu \mathrm{m}$ with a pixel size of 1".4.

(A color version of this figure is available in the online journal.)

\subsubsection{Luminosity Functions}

The luminosity functions of the $\mathrm{H}$ II regions in both galaxies are produced from the $\mathrm{Br} \gamma$ (uncorrected for extinction) images using the IDL program H II phot (Thilker et al. 2000, 2002). $\mathrm{H}$ II phot has an automatic algorithm to identify $\mathrm{H}_{\text {II }}$ region seeds and then grow the $\mathrm{H}_{\text {II }}$ regions from these seeds until it meets another $\mathrm{H}$ II region or reaches a given emission measure gradient. Background subtractions are applied to the identified $\mathrm{H}$ II regions, and both luminosity and $\mathrm{S} / \mathrm{N}$ are measured. The luminosity function of the HII regions detected above $5 \sigma$ are shown for both galaxies, individually and combined, in Figure 4. The luminosity functions all have a power law fit, $d N / d \log L(\operatorname{Br} \gamma) \propto L(\operatorname{Br} \gamma)^{\alpha}$, with power law slope $\alpha$ consistent with -1 (shown in Figure 4), which corresponds to a luminosity function, $d N / d L(\operatorname{Br} \gamma) \propto L(\mathrm{Br} \gamma)^{\alpha-1}$ and agrees with previous studies of $\mathrm{H} \alpha$ luminosity functions (e.g., Kennicutt et al. 1989; Thilker et al. 2000, 2002). The most luminous regions have $L(\mathrm{Br} \gamma) \sim 10^{37.7} \mathrm{erg} \mathrm{s}^{-1}$, corresponding to $L(\mathrm{H} \alpha) \sim 10^{39.7} \mathrm{erg} \mathrm{s}^{-1}$ (if no extinction). These values are also in agreement with previous findings (Thilker et al. 2000, 2002). This provides a consistency check that our continuum subtraction and absolute photometric calibration are reasonable. The $\mathrm{H}_{\mathrm{HI}}$ region luminosity functions have a $5 \sigma$ detection limit of $\log [L(\mathrm{Br} \gamma)] \sim 35.7$, corresponding to star clusters with mass $\sim 4-7 \times 10^{3} M_{\odot}$ (Starburst99; Leitherer et al. 1999), assuming an average age of 4-5 Myr and a fully sampled Kroupa (2001) stellar initial mass function (IMF). Using the estimates of Cerviño et al. (2002) adapted to our IMF, we expect fluctuations on the ionizing photon rate $\delta Q\left(H^{0}\right) / Q\left(H^{0}\right) \lesssim 30 \%$. Thus, our 

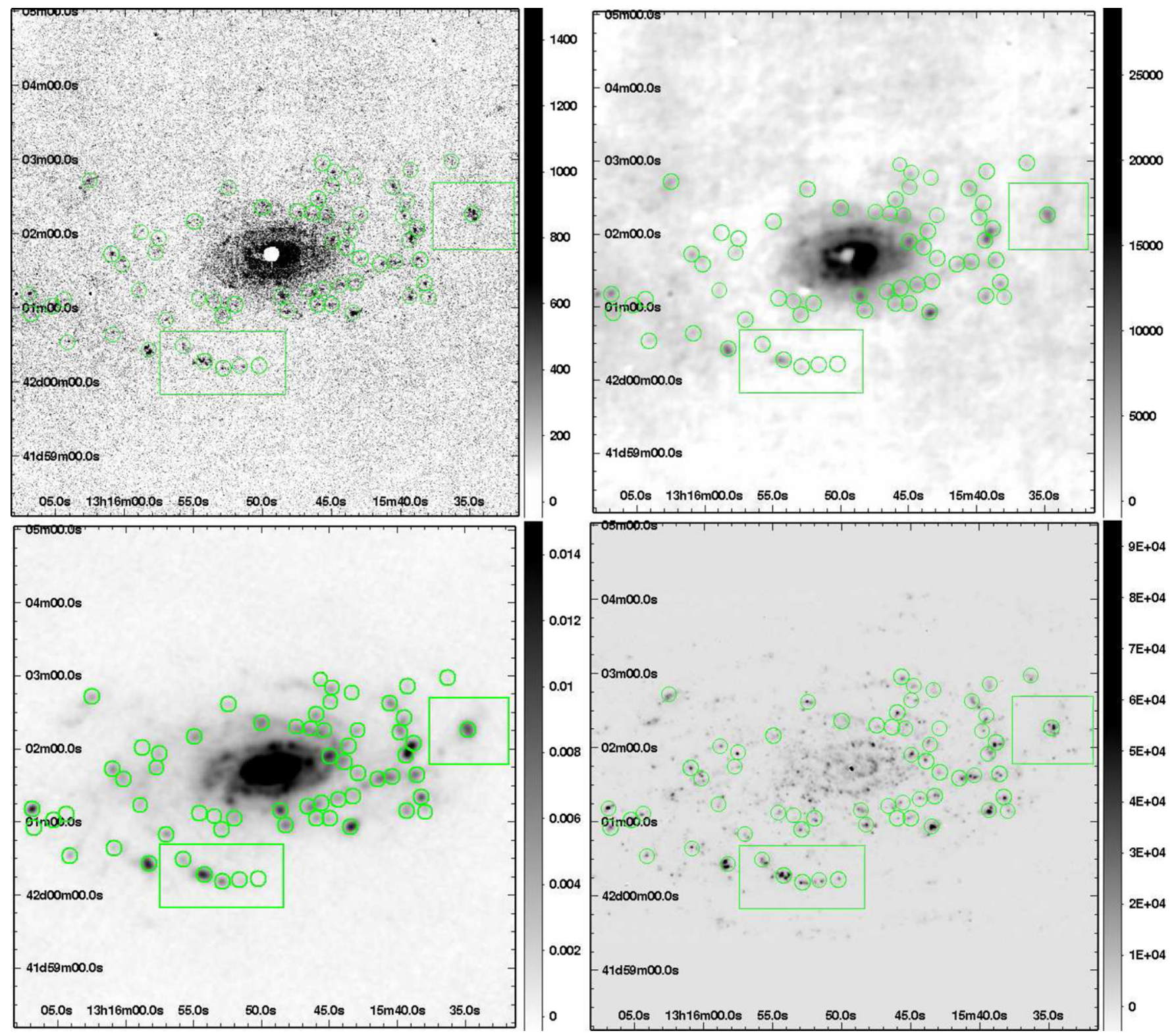

Figure 2. From top left to bottom right: $\mathrm{Br} \gamma, \mathrm{Br} \gamma$ at $70 \mu \mathrm{m}$ resolution, $70 \mu \mathrm{m}$ and $\mathrm{H} \alpha$ images for NGC 5055. The symbols and units for the grayscale bars are the same as described in Figure 1. North is up and east is left. The image size is about $410^{\prime \prime} \times 410^{\prime \prime}$ (about $15.8 \times 15.8 \mathrm{kpc}$ ) for each panel. The uneven background of the $\mathrm{Br} \gamma$ images is greatly enhanced after convolving to the $70 \mu \mathrm{m}$ resolution.

(A color version of this figure is available in the online journal.)

regions encompass a sufficiently large number of young stars that random fluctuations in the ionizing photon flux are not a concern.

\subsubsection{Comparison between $\mathrm{Br} \gamma$ and Free-Free Emission}

To further test our $\mathrm{Br} \gamma$ data, we compare the $\mathrm{Br} \gamma$ emission of NGC 6946 with the thermal free-free component at $33 \mathrm{GHz}$ emission, as observed with the GBT (Murphy et al. 2011b; Figure 5). Murphy et al. (2011b) performed the spectral decomposition of the radio and FIR emission of the targeted regions, separating the free-free emission from other emission components. The GBT observations cover 10 pointings of NGC 6946 (Figure 1 in Murphy et al. 2011b) and have an FWHM about $25^{\prime \prime}$. Our Br $\gamma$ map of NGC 6946 has artifacts in the galaxy center, so we only compare the extranuclear measurements. For our $\mathrm{Br} \gamma$ emission, we also use a $\mathrm{H} \alpha$ map of NGC 6946 for internal extinction correction (see Sections 2.3 and 2.3.1), while the radio emission is insensitive to dust attenuation. Both images are convolved with a Gaussian of FWHM 25" to match the resolution of the GBT $33 \mathrm{GHz}$ observations. The Enuc 4 (the number four extranuclear region as named by Murphy et al. $2011 \mathrm{~b}$ ) is the only region among the 10 observed to have statistically significant excess of anomalous microwave emission in the $33 \mathrm{GHz}$ band (Murphy et al. 2010); however, this region is located outside the coverage of our $\mathrm{H} \alpha$ map, implying that we do not have extinction-corrected $\mathrm{Br} \gamma$ flux for this region. Thus, our comparison between the $\mathrm{Br} \gamma$ and the free-free emission uses a total of eight regions. A test with the extinction correction for the regions before and after the convolution shows less than $5 \%$ difference. As our analysis is on a region by region basis, we adopt the extinction correction after the convolution for our $\mathrm{Br} \gamma$ measurements. Photometry is measured with apertures and background annuli similar to those used by Murphy et al. (2011b). 


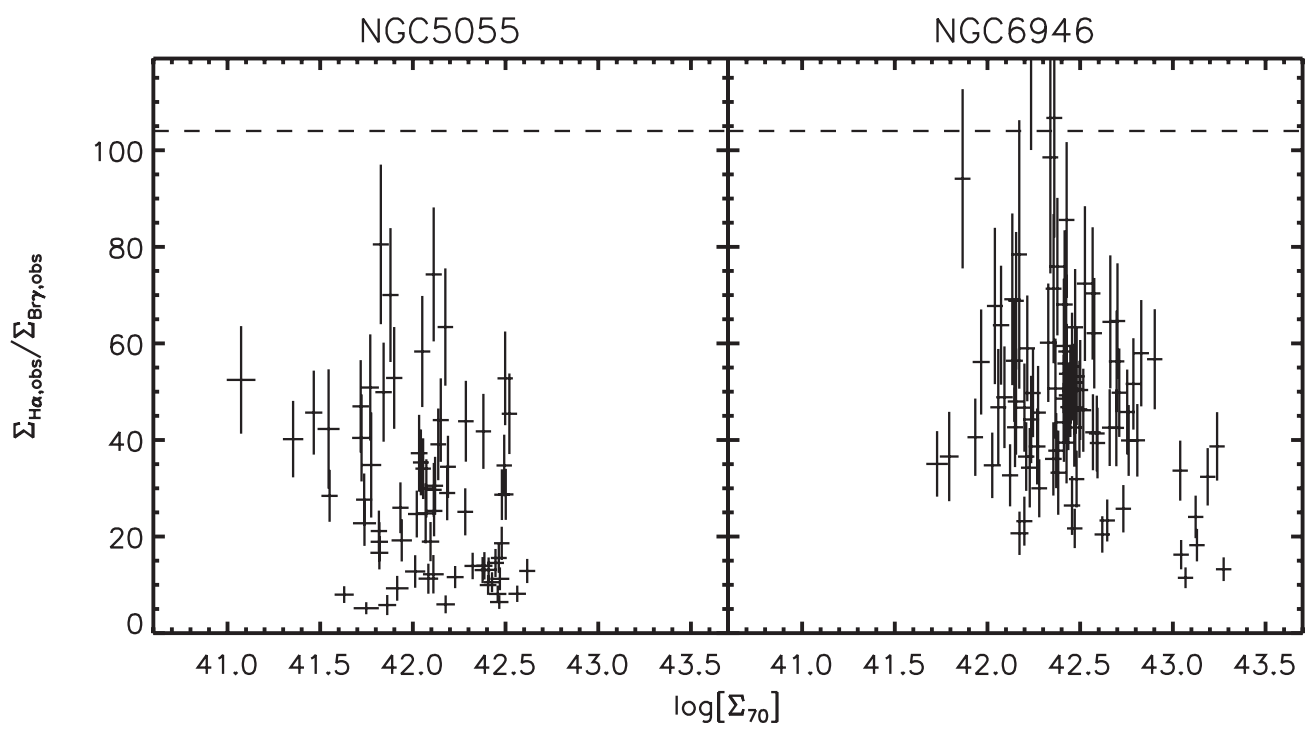

Figure 3. $\Sigma(\mathrm{H} \alpha, \mathrm{obs}) / \Sigma(\mathrm{Br} \gamma$, obs $)$ as a function of $\Sigma(70)$. The two panels are data for NGC 5055 and NGC 6946, respectively, after removal of foreground Galactic extinction. The dashed line indicates the extinction free value of $\Sigma(\mathrm{H} \alpha, \mathrm{obs}) / \Sigma(\mathrm{Br} \gamma, \mathrm{obs})(\sim 103)$. Error bars are shown for each data point. The unit is erg s ${ }^{-1} \mathrm{kpc}^{-2}$ for the LSD.

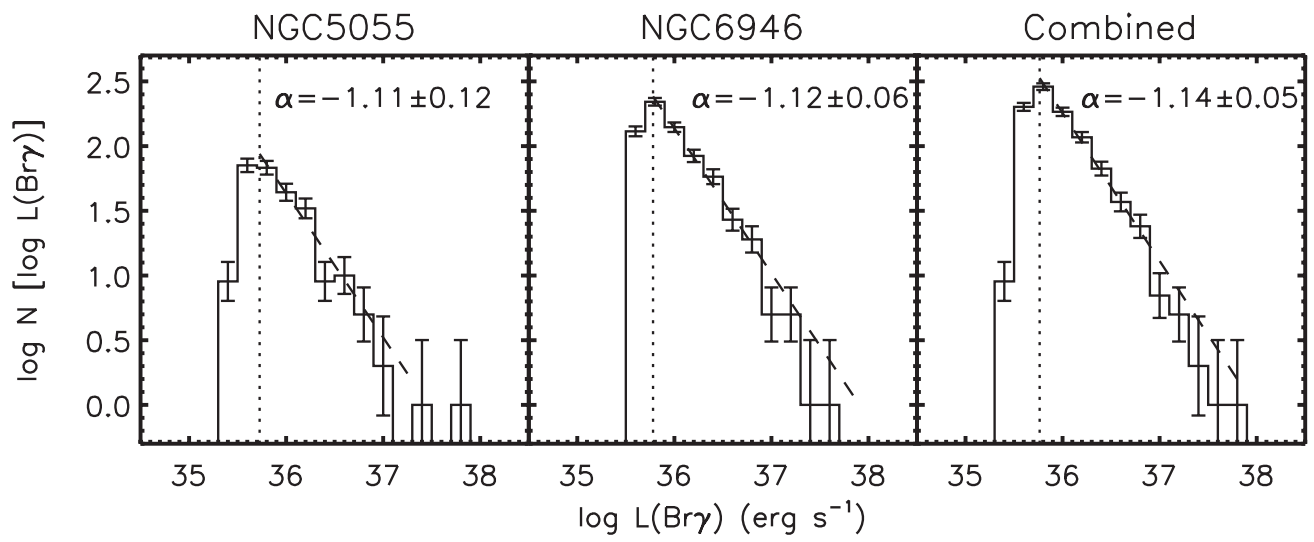

Figure 4. Observed $\operatorname{Br} \gamma$ emission luminosity functions. The vertical dotted lines are $5 \sigma$ detection limit, $\log L(\operatorname{Br} \gamma) \sim 35.7$. The dashed lines are power law fits, $d N / d \log L(\operatorname{Br} \gamma) \propto L(\operatorname{Br} \gamma)^{\alpha}$, for the luminosity functions from the $5 \sigma$ detection limit to the continuous bright end, which are all consistent with $d N / d L(\mathrm{Br} \gamma)$ $\propto L(\mathrm{Br} \gamma)^{-2}$ or $d N / d \log L(\mathrm{Br} \gamma) \propto L(\mathrm{Br} \gamma)^{-1}$.

The galaxy-wide SFR calibrations do not apply precisely to a single-age young populations like those in $\mathrm{H}$ II regions. Here, our apertures correspond to regions consisting of a few $\mathrm{H}$ II regions, and both the free-free and the $\mathrm{Br} \gamma$ measurements are actually reflections of the ionizing photons, $Q\left(H^{0}\right)$, from the young stars. However, for a more direct comparison with previous results, we still calculate the $\mathrm{SFR}(\mathrm{Br} \gamma)$ using the estimated $Q\left(H^{0}\right)$ flux (Osterbrock 1989), with extinction corrected $\mathrm{Br} \gamma$. $\mathrm{SFR}^{T}(33 \mathrm{GHz})$, the SFR derived from the thermal free-free component of the $33 \mathrm{GHz}$ radio emission, is calculated using Equation (11) from Murphy et al. (2011b). Both quantities are calculated assuming electron temperatures of $5000 \mathrm{~K}$ (triangles), 10,000 K (circles), and 20,000 K (squares) to account for the range of variations in electron temperatures found in $\mathrm{H}$ II regions, which depend on galacto-centric radius and metallicity (Shaver et al. 1983). The electron temperature directly affects the intrinsic $\mathrm{H} \alpha / \mathrm{Br} \gamma$ ratio (Osterbrock 1989), thus affecting the extinction correction for the $\mathrm{Br} \gamma$ emission using the $\mathrm{H} \alpha$ emission. It also affects the conversion both from measured luminosities of $\mathrm{Br} \gamma$ and $33 \mathrm{GHz}$ to $Q\left(H^{0}\right)$ flux and from $Q\left(H^{0}\right)$ flux to SFR, as shown in Equation (11) from Murphy et al. (2011b). Values calculated using $\mathrm{Br} \gamma$ emission without internal extinction correction, assuming a typical electron temperature of $10,000 \mathrm{~K}$, are shown as open diamonds, representing about 3\%-18\% corrections for the filled circles in the $\mathrm{Br} \gamma$ emission (with a median $\sim 12 \%$ ) after removal of foreground Galactic extinction. The two inferred SFRs show about $27 \%$ difference on average, for the preferred temperature as shown with the filled symbols. Even when the extreme variations in electron temperature (from $5000 \mathrm{~K}$ to $20,000 \mathrm{~K}$ ) are taken into account, the two inferred SFRs are still in good agreement. This provides again a consistency check that our data reduction and calibration are reliable. Furthermore, our comparison shows that the $\mathrm{Br} \gamma$ data, once corrected for dust extinction using the $\mathrm{H} \alpha$, do not appear to require additional dust corrections, implying that the fraction of ionizing photons completely obscured by dust at $2.16 \mu \mathrm{m}$ is negligible.

Our $3 \sigma$ detection limit within the 12 ".5 radius aperture is $\operatorname{SFR}(\operatorname{Br} \gamma) \sim 0.0017$, or about one order of magnitude fainter than the faintest region analyzed by Murphy et al. (2011b). The higher sensitivity of $\mathrm{Br} \gamma$ imaging suggests that this may become a preferred approach to derive a dust-free SFR indicator over extended sections of star-forming galaxies. 


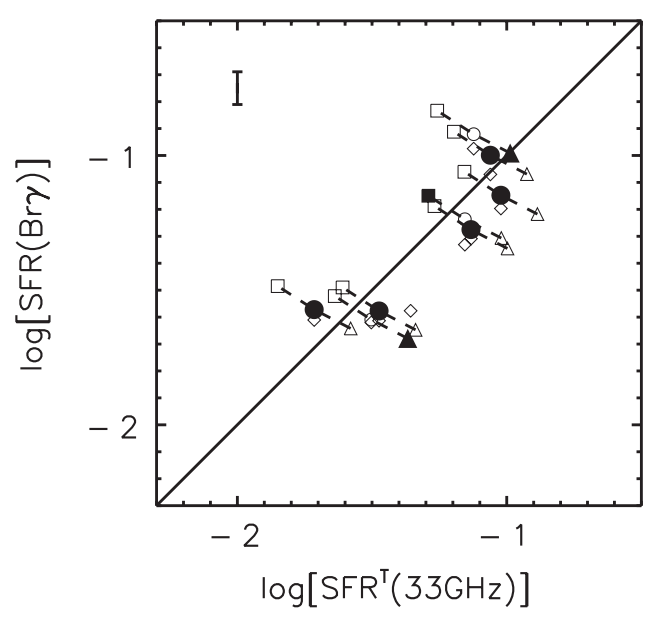

Figure 5. $\operatorname{SFR}(\operatorname{Br} \gamma)$ as a function of SFR derived from thermal free-free emission at $33 \mathrm{GHz}$ (Murphy et al. 2011b). The triangles, circles, and squares are values derived using electron temperatures of 5000, 10,000, and 20,000 K, respectively. The open diamonds are values calculated using $\mathrm{Br} \gamma$ emission without internal extinction correction, assuming a typical electron temperature of $10,000 \mathrm{~K}$. The filled symbols show the preferred temperature for each region as derived in Murphy et al. (2011b). The one open diamond in the middle of the plot without a corresponding filled symbol is for Enuc 4, which we have no coverage with our $\mathrm{H} \alpha$ map thus no extinction corrected value. The typical error bar for $\operatorname{SFR}(\operatorname{Br} \gamma)$ is shown on the upper left corner. The unit is $M_{\odot} \mathrm{yr}^{-1}$ for SFR.

\subsection{PSF-matching}

In order to measure the photometry consistently among the different maps $(\mathrm{H} \alpha, \mathrm{Br} \gamma$ and $70 \mu \mathrm{m})$, we first need to convolve both the $\mathrm{H} \alpha$ and the $\mathrm{Br} \gamma$ maps to the resolution of the $70 \mu \mathrm{m}$ map ( $\left.5^{\prime \prime} .5\right)$. The point-spread function (PSF) FWHM of the $\mathrm{Br} \gamma$ is about $1^{\prime \prime} .1$ and the $\mathrm{H} \alpha$ only slightly better (about $1^{\prime \prime}$ ).

For the PACS PSFs, we choose the observed PSFs of the asteroid Vesta available on the PACS Web site. ${ }^{29}$ We use the PSF with the same scan speed $\left(20^{\prime \prime} \mathrm{s}^{-1}\right)$ and similar orientation (42 deg) to the KINGFISH observations. We measure the curve of growth of the observed PSF and find that more than two-thirds of total flux is included within a radius of $6 "$, slightly larger than the PSF FWHMs, 5".5. Although we think this specific observed PSF should be the best match to our observations, the curve of growth shows less than $1 \%$ difference among observed PSFs with different observational parameters. The model PSFs show a different curve of growth but they still contain more than $2 / 3$ of total flux within the radius of $6^{\prime \prime}$. Thus we choose $6^{\prime \prime}$ as our aperture radius for the $70 \mu \mathrm{m}$ resolution. The PACS PSFs are not spherically symmetric and are dependent on the scan direction, thus the PSFs are rotated by the scan angle (relative to north, as our maps are reprojected with north-up) of our PACS maps. A visual comparison of the rotated PSF and the brightest pointlike sources on the FIR maps of both galaxies shows differences of only a few degrees of rotation. A comparison between the photometry of $\mathrm{Br} \gamma$ maps convolved with one PSF and the same PSF rotated by 60 degrees shows only about $7 \%$ difference.

Finally, the $\mathrm{H} \alpha$ and $\mathrm{Br} \gamma$ maps are convolved with the observed PSF, rotated with the scan angle of the Herschel observation for each galaxy, to produce the $\mathrm{H} \alpha$ and $\mathrm{Br} \gamma$ maps at $70 \mu \mathrm{m}$ resolution. The convolved $\mathrm{H} \alpha$ and $\mathrm{Br} \gamma$ maps are then registered to the same pixel sizes as the $70 \mu \mathrm{m}$ maps with astrometry matched using the brightest sources in the galaxies. Although the PSF FWHM of the Br $\gamma$ is small compared to the $70 \mu \mathrm{m}$, we still convolve the $70 \mu \mathrm{m}$ images with the extracted PSF from

\footnotetext{
29 http://herschel.esac.esa.int/twiki/bin/view/Public/PacsCalibrationWeb
}

several unsaturated bright stars in the CFHT narrowband images to better match the resolution between the two. We are confident that the convolution brings the optical, NIR and $70 \mu \mathrm{m}$ maps to similar resolution and adds a photometric uncertainty no larger than $2 \%$, combining both the difference in observed PSFs with different observation parameters and possible small mismatch of PSF rotation angle. The final PSF FWHM for the $70 \mu \mathrm{m}$ images, the $\mathrm{Br} \gamma$ images and the $\mathrm{H} \alpha$ images is $~ 5^{\prime \prime} 6$.

\subsection{Photometry in Selected Regions \\ 3.3.1. Aperture Photometry}

Star forming regions are selected on the $\operatorname{Br} \gamma$ images at the original resolution, by placing apertures with radii of 6 " centered on the peaks of ionized gas emission. Care is taken so that apertures do not significantly $(<5 \%)$ overlap. Further inspection of the $70 \mu \mathrm{m}$ maps and the degraded $\mathrm{Br} \gamma$ and $\mathrm{H} \alpha$ maps is performed to make sure that each aperture covers at least one $70 \mu \mathrm{m}$ emission peak and is as close as possible to the emission peak both at $70 \mu \mathrm{m}$ and in the emission line images. Sources near the continuum subtraction residuals of bright stars and the galaxy center are not included as to avoid possible contamination from the residuals. The central region of NGC 5055 is identified to have an AGN by Moustakas et al. (2010), and it shows one single bright source in the center covering up a region of about $10^{\prime \prime}$ radius $(\sim 400 \mathrm{pc})$. This region is also avoided when selecting apertures. We selected a total of 73 regions for NGC 5055 and 94 regions for NGC 6946 (Figures 1 and 2). We note that these regions are not the same as those described in Section 3.1.1; H II phot could not be effectively applied to our resolution degraded images, and we resorted to a manual selection. In addition, due to the lower resolution, the regions used for the bulk of our present analysis are larger (and more luminous) than those used for deriving the luminosity functions.

Following the studies of Calzetti et al. (2005), Li et al. (2010), and Liu et al. (2011), local background subtraction is essential for photometric measurements in spatially resolved regions in order to minimize the effect of diffuse IR emission that is not related to current star formation activity. Furthermore, it will serve to mitigate the effects of the uneven background in our Br $\gamma$ maps. We perform the local background subtraction with two methods: one is to identify a local background region for different apertures (Calzetti et al. 2007; Li et al. 2010); the other is to use an annulus outside each aperture not much larger than the aperture radius itself, a method that follows standard practice for photometric measurements. For the first method, we choose two local background regions within each of the two galaxies, with relatively even background on the $\mathrm{Br} \gamma$ map and similar physical conditions, i.e., on the same spiral arm, to ensure that the diffuse IR emission is similar, by visual inspection. For the second method, we adopt an annulus whose inner radius and outer radius are two times and four times the aperture radius, respectively. The width is chosen to be larger than two times the PSF FWHM and is outside of the second Airy ring. Each annulus is thus close enough to its aperture to minimize the effects of the uneven background while still containing enough pixels for a good local background determination and not including significant emission from the region itself. For both methods, the mode is used for the background level due to crowding in some regions. The background levels between the two methods agree with each other with differences of less than several percent when comparisons are performed in uncrowded regions. The results prove that both methods are valid for effectively 
removing the local background in our maps. Due to the uneven background in our $\mathrm{Br} \gamma$ maps, we choose the annulus method, which is more robust against such variations.

The $70 \mu \mathrm{m}$ resolution aperture correction from a 6 " radius to infinity is 1.466 , which is determined using the PACS observed PSFs convolved with the $\mathrm{Br} \gamma$ PSF. The local background subtraction method described above is included in the aperture correction. We use aperture corrections to best recover the true luminosity of each source we select, but they do not affect the analysis, as all the images are degraded to the same resolution. The aperture corrections are only perfectly valid for point sources; however, our sources are usually compact and the underlying extended emission is relatively weak; thus this correction provides a reasonable estimate of the true luminosity of our sources.

\subsubsection{Error Estimate and Signal-to-noise Criteria}

We correct the $\mathrm{H} \alpha$ and $\mathrm{Br} \gamma$ photometry for foreground Galactic extinction (Schlegel et al. 1998; O'Donnell 1994) with a single value (Table 2), which is significant for NGC 6946 as it is close to the Galactic plane, while the Galactic extinction for the FIR bands is negligible. This correction could be a potential source of systematic error if the adopted values are not correct.

The major uncertainty in the photometry is the calibration uncertainty: $10 \%$ at $70 \mu \mathrm{m}, 15 \%$ at $\mathrm{Br} \gamma$, combining both the absolute calibration uncertainty and the continuum subtraction uncertainty, and $10 \%$ at $\mathrm{H} \alpha$ (see Section 2). The uncertainty in the local background level is also included. We take a $2 \%$ uncertainty in the convolution with the observed PSF at different rotation angles for the three bands. Other uncertainties, such as the global background uncertainty, are relatively small. The final photometric uncertainty is usually dominated by the calibration uncertainty; the uncertainty in local background estimate affects the faint regions more than brighter regions.

We accept as "good data" regions with $\mathrm{S} / \mathrm{N}>3$ for all the three images at $70 \mu \mathrm{m}, \mathrm{H} \alpha$ and $\mathrm{Br} \gamma$. The noise is determined using the variation around the mode in the local background annuli of each region. This $\mathrm{S} / \mathrm{N}$ cut removes the faint regions whose $\mathrm{Br} \gamma$ photometry is complicated by the uneven background and uncertainties in the continuum subtraction. Our $\mathrm{S} / \mathrm{N}>3$ cut corresponds to different luminosity cut-offs for different regions, as some of the regions fall in high background noise areas. In our "good data," only five regions have $\mathrm{S} / \mathrm{N}<5$, which are regions in the east and west outskirts of the galaxies (Figures 2 and 1). About $77 \%$ of the regions have $\mathrm{S} / \mathrm{N}$ greater than 10 . The least luminous region has a $L(\mathrm{Br} \gamma)=10^{36.6} \mathrm{erg} \mathrm{s}^{-1}$ with $\mathrm{S} / \mathrm{N}=10.7$, corresponding to a $\mathrm{S} / \mathrm{N}=3$ limit of $L(\mathrm{Br} \gamma)=$ $10^{36.1} \mathrm{erg} \mathrm{s}^{-1}$ in the low background noise area. The lowest $\mathrm{S} / \mathrm{N}$ region $(\mathrm{S} / \mathrm{N}=3.5)$ has a $L(\mathrm{Br} \gamma)=10^{36.7} \mathrm{erg} \mathrm{s}^{-1}$, corresponding to a $\mathrm{S} / \mathrm{N}=3$ limit of $L(\mathrm{Br} \gamma)=10^{36.6} \mathrm{erg} \mathrm{s}^{-1}$ in the high background noise area. We note that the minimum $L(\mathrm{Br} \gamma)=$ $10^{36.6} \mathrm{erg} \mathrm{s}^{-1}$ is about eight times higher than the limit discussed in Section 3.1.1 for the $\mathrm{Br} \gamma$ luminosity function. This difference is explained by the larger apertures we use in this analysis relative to those of Section 3.1.1, which causes blending of $\mathrm{H}$ II regions. The larger apertures are dictated by the larger $70 \mu \mathrm{m}$ PSF than the native Br $\gamma$ PSF. Thus, the same argument about the random sampling of the stellar IMF not being an issue when deriving SFRs applies to our overall analysis, as well. Comparing the luminosity distribution of our selected regions with the luminosity functions derived using $\mathrm{H}$ II phot (see Section 3.1.1), we find a $50 \%$ completeness limit at $L(\mathrm{Br} \gamma) \sim 10^{37} \mathrm{erg} \mathrm{s}^{-1}$. $80 \%$ of the regions we use for the $L(70)-$ SFR analysis have luminosity brighter than this limit, implying that our results are robust relative to completeness issues. For the "good data," we have median uncertainties (and uncertainty ranges) of about $17 \%(15 \%-33 \%)$ in $\mathrm{Br} \gamma, 11 \%(10 \%-30 \%)$ in $\mathrm{H} \alpha$, and $10 \%$ $(10 \%-19 \%)$ in $70 \mu \mathrm{m}$. The final number of regions used in our analysis is 68 for NGC 5055, and 92 for NGC 6946.

\subsubsection{Other Sources of Bias and Uncertainty}

Absorption of Lyman continuum photons (LyC) by dust is a potential source of bias that decreases the luminosity of hydrogen recombination lines and of the free-free continuum. Estimating this effect observationally is challenging, and models are usually used to estimate the impact of this effect. We follow the parameterization of Dopita et al. (2003), where the lost fraction of $\mathrm{H} \beta$ luminosity to LyC absorption by dust is modeled as the product of the oxygen abundance and ionization parameter. For the typical oxygen abundance (about solar) and ionization parameter range $\left(U \sim 10^{-3}\right.$ ) expected in our regions, we estimate the LyC absorption by dust to be at the level of $10 \%$ or less. Hunt \& Hirashita (2009); Draine (2011) suggests similar scenarios except in very extreme conditions. We thus neglect this small negative contribution in our analysis.

Leakage of ionizing photons out of the regions is also a potential source of bias. Many studies (e.g., Ferguson et al. 1996; Thilker et al. 2002; Oey et al. 2007) place the fraction of ionizing photons escaping from $\mathrm{H}$ II regions at the $40 \%-60 \%$ level, as evaluated from the observed $\mathrm{H} \alpha$. When accounting for the differential dust attenuation between $\mathrm{H}$ II regions and the diffuse medium, the fraction of diffuse $\mathrm{H} \alpha$ is reduced by roughly a factor two (Crocker et al. 2013). We thus expect that our Br $\gamma$ measurements will be systematically biased low compared to the number of ionizing photons produced by about $20 \%-30 \%$. The dependence of this bias on the region's characteristics (luminosity, density, etc.) is still under investigation, with different conclusions reached by different authors (Pellegrini et al. 2012; Crocker et al. 2013). Thus, although the systematic $\sim 20 \%-30 \%$ bias is likely to be present for all or most of our regions, we will not attempt to correct for it, in light of the uncertainty just discussed.

\section{RESULTS AND DISCUSSIONS}

\section{1. $\operatorname{SFR}(H \alpha+70)$}

Calzetti et al. (2007) and Kennicutt et al. (2007, 2009) proposed to use a combination of $24 \mu \mathrm{m}$ emission and $\mathrm{H} \alpha$ emission as an "unbiased" SFR indicator, where the $24 \mu \mathrm{m}$ emission is used to represent the attenuated SFR, i.e., attenuated $\mathrm{H} \alpha$ emission. Following these studies, we compare the extinction correction and the $70 \mu \mathrm{m}$ emission, to see if a similar combination,

$$
\Sigma(\mathrm{H} \alpha, \mathrm{corr})=\Sigma(\mathrm{H} \alpha, \text { obs })+\alpha \Sigma(70)
$$

can be established using the $70 \mu \mathrm{m}$ emission instead of the $24 \mu \mathrm{m}$ emission, where corr stands for extinction corrected. Figure 6 shows $\Sigma(70) / \Sigma(\mathrm{H} \alpha$, obs $)$ as a function of $10^{0.4 A(\mathrm{H} \alpha)}-1$, since

$\alpha=\frac{\Sigma(\mathrm{H} \alpha, \text { corr })-\Sigma(\mathrm{H} \alpha, \text { obs })}{\Sigma(70)}=\frac{\left(10^{0.4 \mathrm{~A}(\mathrm{H} \alpha)}-1\right) \Sigma(\mathrm{H} \alpha, \mathrm{obs})}{\Sigma(70)}$.

Linear fits in $\log -\log$ space, $\log [\Sigma(70) / \Sigma(\mathrm{H} \alpha$, obs $)]=\mathrm{a}_{0}+$ $\mathrm{b}_{0} \log \left[10^{0.4 \mathrm{~A}(\mathrm{H} \alpha)}-1\right]$ and linear fits with fixed unity slope in $\log -\log$ space, $\log [\Sigma(70) / \Sigma(\mathrm{H} \alpha$, obs $)]=a_{1}+\log \left[10^{0.4 A(\mathrm{H} \alpha)}-1\right]$, 


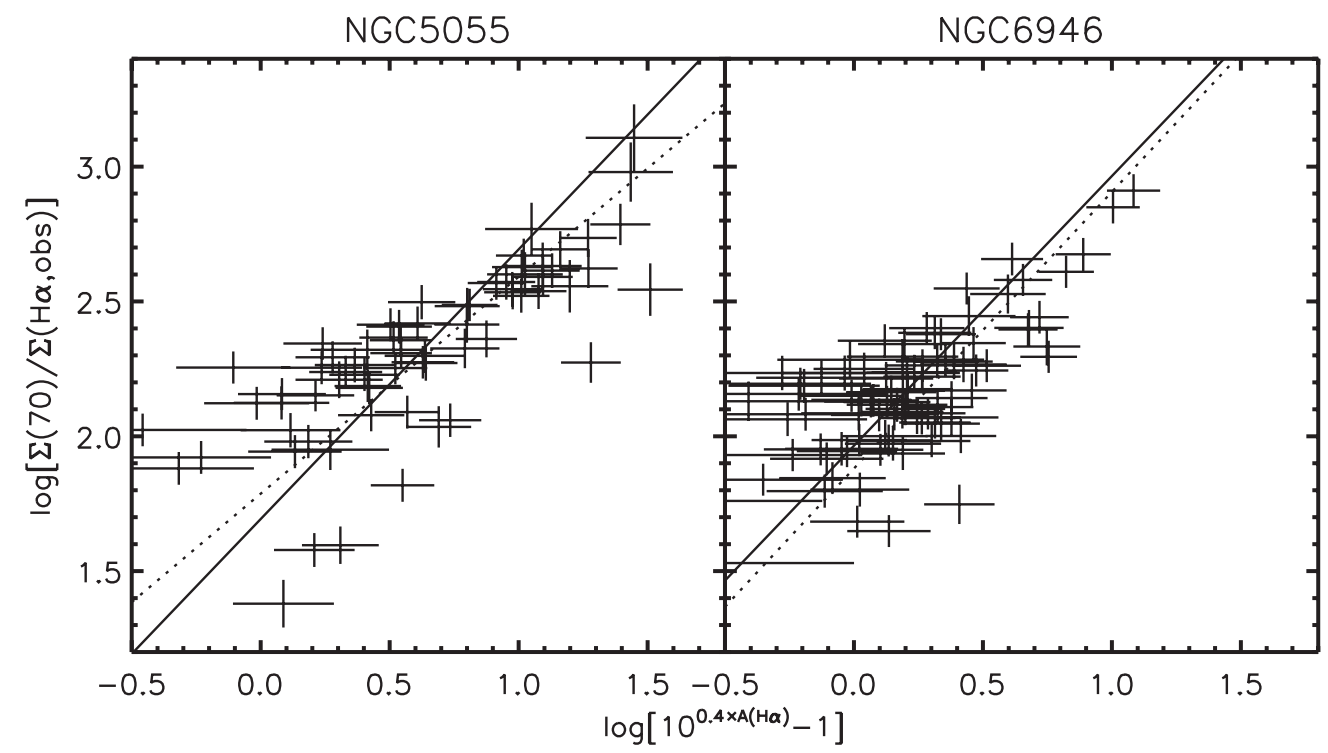

Figure 6. Ratio of $\Sigma(70)$ to $\Sigma\left(\mathrm{H} \alpha\right.$, obs) as a function of $10^{0.4 A(\mathrm{H} \alpha)}-1$ (representing the attenuated fraction of $\mathrm{H} \alpha$ emission, see Section 4.1). The two plots are data for NGC 5055 and NGC 6946, respectively. Dotted lines are linear fits in log-log space and solid lines are linear fit with unity slope in log-log space.

Table 4

Fitting Results: $\log [\Sigma(70) / \Sigma(\mathrm{H} \alpha$, obs $)]$ versus $\log \left[10^{0.4 A(\mathrm{H} \alpha)}-1\right]$

\begin{tabular}{|c|c|c|c|c|c|}
\hline \multirow[b]{2}{*}{ (1) } & \multicolumn{3}{|c|}{ Fitting Parameters } & \multirow[b]{2}{*}{$\begin{array}{c}\alpha \\
(5)\end{array}$} & \multirow[b]{2}{*}{$\begin{array}{c}\sigma \\
(6)\end{array}$} \\
\hline & $\begin{array}{l}a_{0} \\
(2)\end{array}$ & $\begin{array}{l}b_{0} \\
(3)\end{array}$ & $\begin{array}{l}a_{1} \\
\text { (4) }\end{array}$ & & \\
\hline NGC 5055 & $1.787(0.034)$ & $0.804(0.049)$ & $1.693(0.021)$ & $\ldots$ & 0.23 \\
\hline NGC 6946 & $1.881(0.027)$ & $1.026(0.077)$ & $1.965(0.024)$ & $0.011(0.001)$ & 0.24 \\
\hline
\end{tabular}

Notes. Column 1: galaxy; Columns 2-4: fit parameters as in $y=a_{0}+b_{0} x$ and $y=a_{1}+x$; Column 5: derived $\alpha$ assuming $\Sigma_{\mathrm{H} \alpha, \text { corr }}=\Sigma_{\mathrm{H} \alpha, \text { obs }}+\alpha \Sigma_{70}$ with $\alpha=$ $1 / 10^{a_{1}}$ (see Section 4.1); Column 6: $1 \sigma$ dispersion of the data around the linear fit.

are shown as dotted lines and solid lines, respectively, and the fit parameters are listed in Table 4. The best-fit slope of NGC 6946 is consistent with unity and if we adopt the forced fit with unity slope, we obtain $\alpha=1 / 10^{a_{1}}$, which is also listed in Table 4 . The $1 \sigma$ dispersions of the data around the linear fit are also listed; these dispersions provide a better measure of the accuracy of the linear fit, as the formal uncertainties listed in Table 4 (and subsequent tables) reflect only the internal dispersion resulting from the fit procedure. We use the IDL routine "fitexy" to perform the fit process here and for later fits. This takes into account the uncertainties in both axes and derives a straight line fit using $\chi$-square minimization.

We further test the robustness of our fits, as it seems that the linear fit is determined by the highest four data points and thus larger uncertainty may occur without those data points, for the NGC 6946 fit. Though there is no physical reason to not include these points, we obtain a fit slope of $1.062( \pm 0.111)$ if we exclude them from the fit, which is not too much different from the original fit slope $1.026( \pm 0.077)$ (Table 4) and the fit uncertainty is only slightly increased. Thus, it reveals that our fit is actually robust.

The linear trend obtained for NGC 6946 indicates that using the PACS $70 \mu \mathrm{m}$ band as a complementary SFR tracer to correct for the attenuated $\mathrm{H} \alpha$ emission is a viable method, with a corresponding $\alpha$ value of $0.011( \pm 0.001)$. We obtain a best-fit slope significantly smaller than unity for NGC 5055, probably

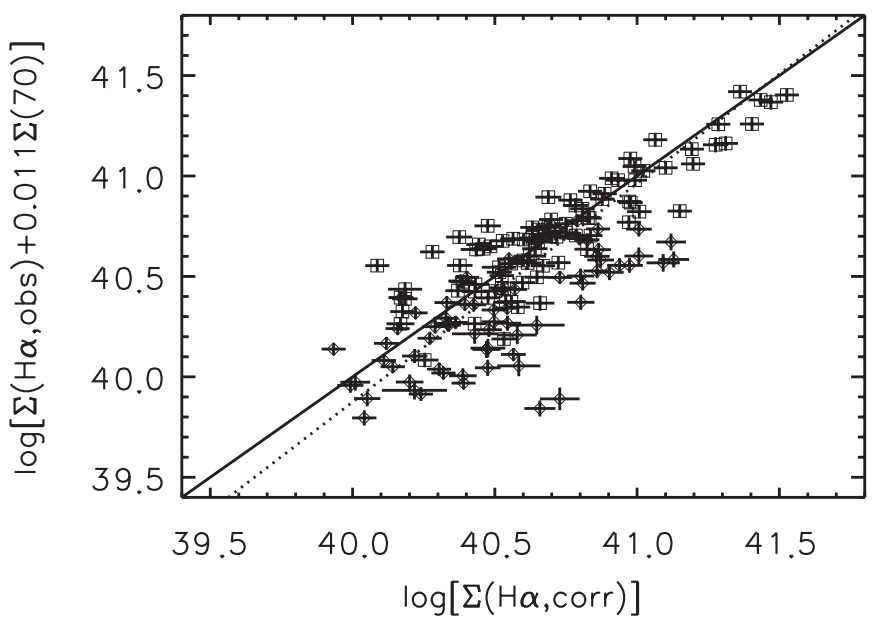

Figure 7. $\Sigma(\mathrm{H} \alpha, \mathrm{obs})+0.011 \Sigma(70)$ as a function of extinction corrected $\Sigma(\mathrm{H} \alpha)$. The open diamonds and open squares are data for NGC 5055 and NGC 6946, respectively. The dotted line is a linear fits in log-log space and the solid line is the one-to-one line. The linear fit slope is 1.09(0.02). The dispersion in the data around the linear fit is 0.22 dex. The unit is erg s $\mathrm{s}^{-1} \mathrm{kpc}^{-2}$ for LSD.

due to its larger inclination (see Section 4.4). To test the applicability of the derived $\alpha$, we plot $\log [\Sigma(\mathrm{H} \alpha$, obs $)+0.011 \Sigma(70)]$ as a function of $\log [\Sigma(\mathrm{H} \alpha$, corr $)]$, the extinction corrected $\mathrm{H} \alpha$ LSD (Figure 7), for these two galaxies. The general trend of the data is close to the one-to-one correlation, indicating that $\alpha=0.011$ works on average. However, the NGC 5055 data lie systematically below the one-to-one correlation. This deviation is discussed in more detail in the next section. A comparison of this derived $\alpha=0.011$ to the proportionality parameter for $\mathrm{H} \alpha+\alpha \times 24 \mu \mathrm{m}(\alpha=0.031$; Calzetti et al. 2007), gives a ratio $L(70) / L(24) \sim 3$, which is consistent with the model predictions by Draine \& Li (2007) with an average stellar radiation field strength $U$ about 300 , the value estimated for star-forming regions (Calzetti et al. 2007). The current sample size is too small to enable a detailed comparison between the 24 micron and 70 micron mixed indicators, which we defer to a later paper where a larger number of galaxies will be analyzed. 


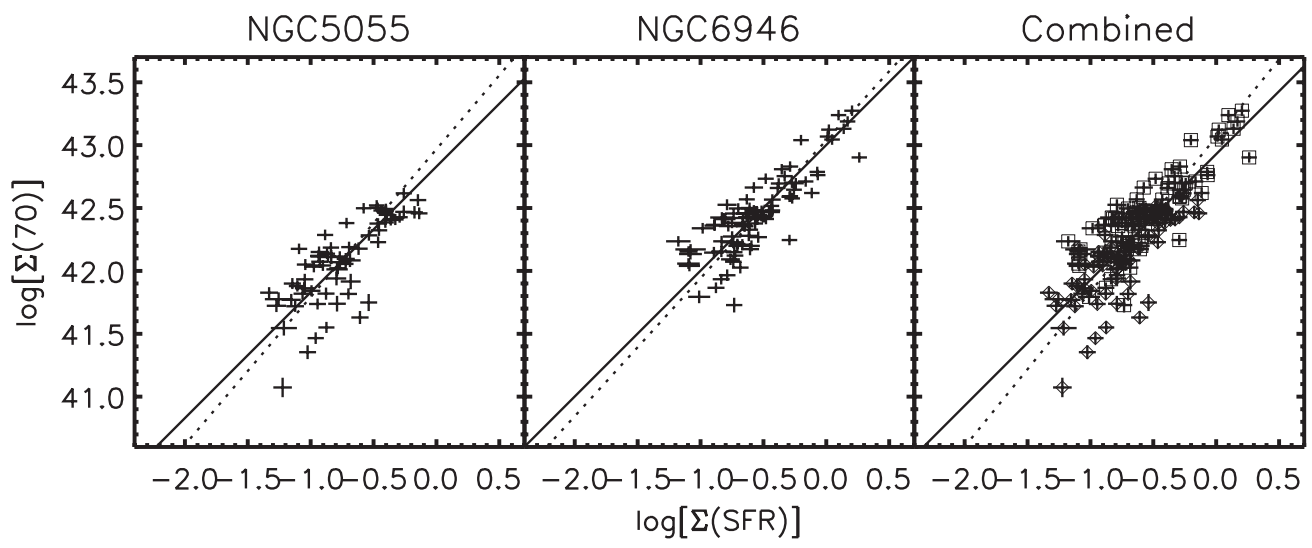

Figure 8. $\Sigma(70)$ as a function of $\Sigma(\mathrm{SFR})$, from extinction corrected $\Sigma(\mathrm{Br} \gamma)$. The three columns are data for NGC 5055, NGC 6946 and both combined, respectively. In the third column, open diamonds are for NGC 5055 and open squares are data for NGC 6946. Dotted lines are linear fits in log-log space and solid lines are linear fit with unity slope in $\log -\log$ space. The units are $M_{\odot} \mathrm{yr}^{-1} \mathrm{kpc}^{-2}$ for SFR surface density and erg s${ }^{-1} \mathrm{kpc}^{-2}$ for LSD.

\section{2. $\operatorname{SFR}(70)$}

In Section 4.1 and in this section we seek to obtain a linear trend between the sum of $\mathrm{H} \alpha$ plus the $70 \mu \mathrm{m}$ emission and $\mathrm{Br} \gamma$ and between the $70 \mu \mathrm{m}$ emission and $\mathrm{Br} \gamma$, respectively. At first sight, it will appear that seeking linear trends (slope of unity in a log-log plot) in both cases is contradictory. However, we note that the range of luminosities for which each calibration is valid will be different and only partially overlapping (see, e.g., discussion in Kennicutt et al. 2009; Calzetti et al. 2010). The $70 \mu \mathrm{m}$ emission will generally be stronger in dust obscured regions where $\mathrm{H} \alpha$ emission is weak.

We express the IR (70, 100, and $160 \mu \mathrm{m}$ bands) SFR calibrations in this paper as

$$
\begin{aligned}
\operatorname{SFR}(\operatorname{IR} \text { band })\left(M_{\odot} \mathrm{yr}^{-1}\right)= & \mathcal{C}_{\mathrm{IR} \text { band,region }} \\
& \times 10^{-43} \times \mathrm{L}(\mathrm{IR} \text { band })\left(\mathrm{erg} \mathrm{s}^{-1}\right) .
\end{aligned}
$$

We refer to the $\mathcal{C}$ as the Calibration Coefficient. Thus, the Calibration Coefficients in Calzetti et al. (2010) and Li et al. (2010) are $\mathcal{C}_{70, \text { galaxy }}=0.58$, and $\mathcal{C}_{70,700 \mathrm{pc}}=0.94$, respectively. Using the $70 \mu \mathrm{m}$ to the bolometric IR luminosity ratio, Lawton et al. (2010) has derived $\mathcal{C}_{70,10-300 \mathrm{pc}}=0.97$ for the Magellanic Clouds.

Adopting SFR $=5.45 \times 10^{-42} \times L(\mathrm{H} \alpha)($ Calzetti et al. 2010) and $L(\mathrm{H} \alpha)=103 \times L(\mathrm{Br} \gamma)$, we derive SFRs for our regions using their extinction corrected $\mathrm{Br} \gamma$ luminosities. Figure 8 shows the $\Sigma(70)$ as a function of $\Sigma(\mathrm{SFR})$. The linear fits in $\log -\log$ space, $\log [\Sigma(70)]=a_{0}+b_{0} \log [\Sigma(\mathrm{SFR})]$, and the fixed unity slope fits, $\log [\Sigma(70)]=a_{1}+\log [\Sigma($ SFR $)]$, are shown as dotted lines and solid lines, respectively, and the fit parameters are listed in Table 5. The $1 \sigma$ dispersions of the data around the linear fit are also listed. The fits all have slopes close to unity and so we adopt the fit results with unity slope to obtain the Calibration Coefficients, $\mathcal{C}=10^{43-a_{1}}$, used in Equation (4). These are also listed in Table 5 ; the $\mathcal{C}_{70,200 \mathrm{pc}}=1.18(0.02)$ for the combined data (at $\sim 200$ pc scale) will be used for the comparison with the Calibration Coefficients, $\mathcal{C}_{70}$, from Calzetti et al. (2010) and Li et al. (2010), as larger samples of galaxies were combined to derive the calibration in those works.

The calibration coefficient of NGC 5055 is higher in value than that of NGC 6946 (Table 4). We provide analysis with the longer wavelengths and reasons in Section 4.4 that lead us to believe that the anomalous behavior is in NGC 5055. We suggest
Table 5

\begin{tabular}{|c|c|c|c|c|c|}
\hline \multirow[b]{2}{*}{ (1) } & \multicolumn{3}{|c|}{ Fitting Parameters } & \multirow[b]{2}{*}{$\begin{array}{l}\mathcal{C}_{70} \\
(5)\end{array}$} & \multirow[b]{2}{*}{$\begin{array}{c}\sigma \\
(6)\end{array}$} \\
\hline & $\begin{array}{l}a_{0} \\
(2)\end{array}$ & $\begin{array}{l}b_{0} \\
\text { (3) }\end{array}$ & $\begin{array}{l}a_{1} \\
\text { (4) }\end{array}$ & & \\
\hline NGC 5055 & $42.967(0.037)$ & $1.176(0.047)$ & $42.829(0.010)$ & $1.48(0.04)$ & 0.24 \\
\hline NGC 6946 & $43.038(0.020)$ & $1.097(0.033)$ & $43.001(0.009)$ & $1.00(0.02)$ & 0.19 \\
\hline Combined & $43.065(0.019)$ & $1.232(0.027)$ & $42.928(0.007)$ & $1.18(0.02)$ & 0.23 \\
\hline
\end{tabular}

Fitting Results: $\log [\Sigma(70)]$ versus $\log [\Sigma(\mathrm{SFR})]$

Notes. Column 1: data set; Columns 2-4: fit parameters as in $y=a_{0}+b_{0} x$ and $y=a_{1}+x$; Column 5: derived Calibration Coefficient, $\mathcal{C}_{70}=10^{43-a_{1}}$; Column 6: $1 \sigma$ dispersion of the data around the linear fit.

that the higher inclination of NGC 5055 relative to NGC 6946 prevents an effective removal of the diffuse dust along the line of sight to the H II regions of the galaxy and/or causes oversubtraction of the star forming regions contributing to the local 70 micron emission. Although we use circular annuli around each region to remove the foreground/background diffuse component of dust emission, the high inclination of NGC 5055 implies that there is more intervening dust emission within each photometric aperture that is not directly related to the H II region(s), and removal of such a component is subject to larger systematic uncertainties. Indeed, as we show in Section 4.4, going to lower resolution (longer wavelengths) we increase lineof-sight confusion and the NGC 5055 data further deviate from those of NGC 6946. Draine et al. (2007) show that the mean interstellar radiation field is 2.5 times weaker in NGC 5055 than in NGC 6946, which also manifests itself as a global IR spectral energy distribution (SED) that has a slightly colder mean temperature (Skibba et al. 2011; Dale et al. 2012). Thus, the intervening diffuse component in the NGC 5055 apertures is made of dust that is slightly colder than that in NGC 6946, which accounts for the behavior observed in Section 4.4 at 100 and $160 \mu \mathrm{m}$. In addition to the possible over-subtraction, this also helps account for the higher calibration coefficient in NGC 5055 than in NGC 6946: the peak of the diffuse dust emission in the former galaxy is shifted to longer wavelengths than in the latter. In fact, $\mathrm{H}$ II regions in NGC 5055 show higher extinction values than in NGC 6946 (Figure 3), which may imply higher selfshielding for the dust surrounding the $\mathrm{H}$ II regions, resulting in cooler mean dust temperatures (e.g., Calzetti et al. 2010). Both effects are likely to contribute to the $\sim 50 \%$ higher $\mathcal{C}_{70,200 \mathrm{pc}}$ in NGC 5055. 
We also revisit the data for both NGC 5055 and NGC 6946 in $\mathrm{Li}$ et al. (2010) to understand the $\sim 50 \%$ higher $\mathcal{C}_{70,200 \mathrm{pc}}$ of NGC 5055. We find that the NGC 5055 data in that work already show a $2 \sigma$ deviation from the mean trend in the sense of being systematically higher than the mean, while the NGC 6946 data lie about the mean trend. Indeed, the corresponding $\mathcal{C}_{70,700 \mathrm{pc}}$ value of NGC 5055 is also about $50 \%$ larger that that of NGC 6946 in the data of Li et al. (2010). This strongly suggests that a larger sample with more galaxies is needed to average out fluctuations and derive a more reliable general calibration.

Although we find roughly linear trends both in Section 4.1 and this section, we should be reminded that our study only involves two galaxies, and uncertainties are large. A study involving a larger sample (which we are planning in the near future) will be better suited for analyzing similarities and differences in the two calibrations.

\subsection{SFR Calibrations at Different Scales}

Li et al. (2010) found, from a comparison of the Calibration Coefficients between $70 \mu \mathrm{m}$ emission in sub-galactic regions $\left(\mathcal{C}_{70,700 \mathrm{pc}}\right)$ and for integrated (star-forming and starburst) galaxies $\left(\mathcal{C}_{70 \text {,galaxy }}\right)$, a $\sim 40 \%$ difference between the two values, which they attributed to dust heated by stellar populations not related to the current star formation activity. The comparison is performed using the following steps: for the calibration in Calzetti et al. (2010), a galaxy with a SFR of $1 M_{\odot} \mathrm{yr}^{-1}$ implies a $70 \mu \mathrm{m}$ luminosity of $1.725 \times 10^{43} \mathrm{erg} \mathrm{s}^{-1}$; the calibration of Li et al. (2010) shows that $\sim 700 \mathrm{pc}$ sub-galactic star forming regions with the same total SFR of $1 M_{\odot} \mathrm{yr}^{-1}$ have a total $70 \mu \mathrm{m}$ luminosity of only $1.067 \times 10^{43} \mathrm{erg} \mathrm{s}^{-1}$; thus, the difference in these two calibrations reveals an average of $\sim 40 \%$ excess $70 \mu \mathrm{m}$ emission in the galaxies on scales larger than $700 \mathrm{pc}$. With the results in Table 5, we add another $\mathcal{C}_{70}$, derived from the two galaxies, with an average region size of $\sim 210$ pc (Figure 9 ). $\mathcal{C}_{70}$ increases as we probe star forming regions with smaller size, which gives a factor two difference between the 200 pc calibration and the whole galaxy calibration, implying that $\sim 50 \%$ of the $70 \mu \mathrm{m}$ emission beyond the $200 \mathrm{pc}$ scale is due to heating of dust by stars not associated with current star formation.

In the comparison above, we assume that the dust heated by young stellar populations in whole galaxies is a scaled-up version of dust heating in $200 \mathrm{pc}$ regions, as traced by the ionizing photon flux. This is equivalent to assuming the UV photons leaked from the $\mathrm{H}$ II regions trace the leakage of ionizing photons, and that this ratio is constant over all spatial scales. The leakage of ionizing photons out of $\mathrm{H}$ II regions is not included in our estimate of the $\Sigma$ (SFR). If we were to systematically correct all the Br $\gamma$ luminosities by $\sim 30 \%$ (Section 3.3.3), the estimate of the diffuse fraction as inferred from the comparison between $\mathcal{C}_{70,200 \mathrm{pc}}$ and $\mathcal{C}_{70 \text {,galaxies }}$ would increase by a similar percentage, from $\sim 50 \%$ to $\sim 65 \%$. This new estimate, however, does not include the leakage of dust heating UV photons from the H II regions; if we assume, as done so far, that they trace the leakage of ionizing photons, the estimate of the diffuse $70 \mu \mathrm{m}$ fraction is reset back to $\sim 50 \%$.

Lawton et al. (2010) provide estimates for the size of H II regions in the IR. Their analysis yields mean $70 \mu \mathrm{m}$ radii of $60( \pm 20)$ pc for HiI regions in the Large Magellanic Cloud (LMC) and $80( \pm 30) \mathrm{pc}$ for H II regions in the Small Magellanic Cloud (SMC). The radii are calculated as encompassing $95 \%$ of the light. Our $\sim 200 \mathrm{pc}$ radii apertures are thus likely to include most of the UV photons responsible for heating the dust within and around $\mathrm{H}$ II regions; the $50 \%$ estimate for the diffuse fraction outside $200 \mathrm{pc}$ radii is thus likely to be a conservative one.

The interpretation we give for the trend in Figure 9 is that smaller regions contain smaller fractions of diffuse IR emission heated by stellar populations that is not related to current star formation, i.e., populations older than about 10 Myr. We make use of a simple model ( $\mathrm{Li}$ et al. 2010) to try to qualitatively support this interpretation. The simple model involves using (1) Starburst99 to produce a stellar SED for a given star formation history; (2) the attenuation curve from Calzetti et al. (2000) applied to the stellar light to produce the total IR emission; (3) the dust model from Draine \& Li (2007) to predict IR emission in a given band (here $70 \mu \mathrm{m}$ only). We adopt continuous star formation populations over the three timescales of $100 \mathrm{Myr}, 1 \mathrm{Gyr}$ and $10 \mathrm{Gyr}$ and derive $\mathcal{C}_{70}$ for each timescale. The applied attenuation value is the average of the two values from Table 2 . We then place the three model $\mathcal{C}_{70}$ values in Figure 9 (star symbols). The physical scales are assigned to the models based on an assumed region crossing speed of stars of $v \sim 3 \mathrm{~km} \mathrm{~s}^{-1}$ (in agreement with typical velocity dispersions of young stars; Sotnikova \& Rodionov 2003), a proxy that relates physical scales to timescales. From the crude model comparison, one can tell that the $200 \mathrm{pc}$ calibration (filled circle) is close to the 100 Myr model while the whole galaxy calibration (filled square) is close to a $10 \mathrm{Gyr}$ model. In fact, a longer star formation timescale accumulates more stars with long lifetimes, which will heat the dust. The contribution to the IR emission from these stars will need to be removed from the SFR accounting, resulting in a smaller calibration coefficient for whole galaxies than for small regions.

At high surface brightness values, the spatially resolved PACS measurements tend to be higher than the MIPS measurements at the same wavelength, which has been attributed to the onset of nonlinear behavior in the MIPS detectors (see, e.g., the photometric experiments by the PACS team ${ }^{30,31}$ ). If our MIPS $70 \mu \mathrm{m}$ measurements in the $700 \mathrm{pc}$ regions are, indeed, slightly underestimated, we would need to increase the measured fluxes at this spatial scale, in order to bring them to a consistent flux scale with the $200 \mathrm{pc}$ measurements. As a result, in order to recover the correct SFR at $700 \mathrm{pc}$, the calibration constant would need to be decreased, which would only increase our already found discrepancy between the 200 pc calibration and the $700 \mathrm{pc}$ calibration. In reality, we expect the effect of the discrepancy between PACS and MIPS responses at high surface brightnesses to be small on $700 \mathrm{pc}$ scales, since the whole galaxy PACS $70 \mu \mathrm{m}$ measurement is not significantly different from the MIPS one (Dale et al. 2012). Thus our estimated diffuse $70 \mu \mathrm{m}$ fraction of the total emission, as derived by comparing the $200 \mathrm{pc}$ scale to the integrated calibrations, is likely to be an accurate estimate.

The open circle in Figure 9 is the Calibration Coefficient derived from the same combined data sets of NGC 5055 and NGC 6946, without the local background subtracted from the $70 \mu \mathrm{m}$ photometry. The open diamond is the Calibration Coefficient for $\sim 700 \mathrm{pc}$ regions also without the local background subtracted. Simply by comparing the filled circle and diamond with the open circle and diamond, we obtain about $31 \%$ and $22 \%$ of diffuse emission in the $70 \mu \mathrm{m}$ IR bands when comparing whole galaxies with $200 \mathrm{pc}$ and $700 \mathrm{pc}$ regions, respectively. Thus, even when the local background is not subtracted from

\footnotetext{
30 https://nhscsci.ipac.caltech.edu/pacs/docs/Photometer/ PICC-NHSC-TR-034.pdf

31 http://herschel.esac.esa.int/twiki/pub/Public/PacsCalibrationWeb/ ExtSrcPhotom.pdf
} 


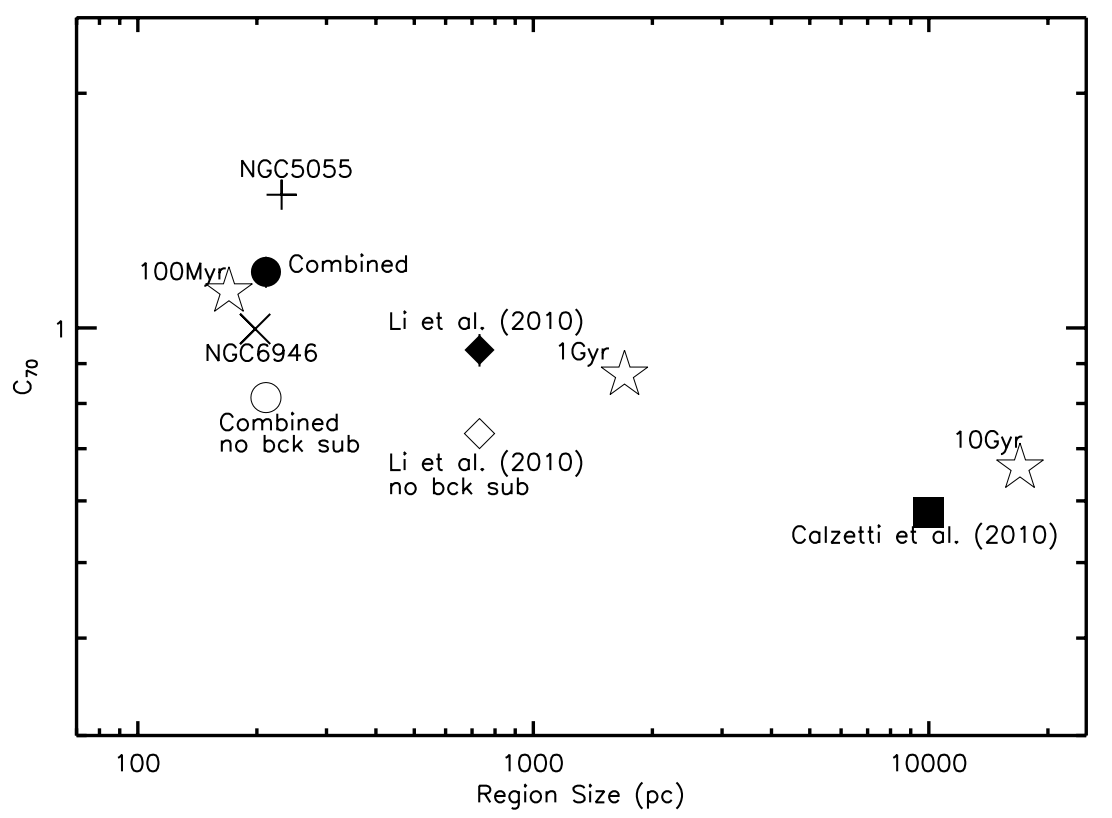

Figure 9. Calibration Coefficients of the $70 \mu \mathrm{m}$ band $\left(\mathcal{C}_{70}\right)$ as a function of region physical size. The plus symbol is for NGC 5055 and the cross symbol is for NGC 6946, using the values derived in the present work. The filled circle is for both galaxies combined (with a combined sample of 160 regions). The filled diamond is from Li et al. (2010, using 556 regions). The filled square is from Calzetti et al. (2010, using 189 star-forming and starburst galaxies). For all data with filled symbols, $3 \sigma$ error bars are shown (the filled square is larger than its error bar). The open circle is for the two galaxies in our work combined, but without the local background removed from the $70 \mu \mathrm{m}$ photometry. Similarly the open diamond is for the $700 \mathrm{pc}$ regions of Li et al. (2010) without the local background removed. The three star symbols are $\mathcal{C}_{70}$ determined for continuous star formation populations with different timescales, using a simple model; they are placed on the plot after associating physical scales to their duration of star formation (see the text).

the $200 \mathrm{pc}$ and $700 \mathrm{pc}$ apertures, we still find significant diffuse emission in the whole galaxy, and its fraction still increases with decreasing aperture size (Figure 9). The persistency of the trend with aperture size even in the absence of local background subtraction suggests that the diffuse emission contribution in galaxies is significant in the IR bands and supports our choice to remove the local background in our analysis. Our choice of local background subtraction, by using annuli as close as possible to the aperture, is generally more robust than other methods (e.g., large regions backgrounds, etc.) against background variations, both real and spurious. Thus, we consider our results representative of the trends we should recover in other galaxies, as well. However, the local background subtraction is still limited by the resolution and the diffuse contribution associated with scales smaller than the resolution can not be or at least can not be effectively removed, thus producing the differences between the 200 and $700 \mathrm{pc}$ analysis.

To summarize, with the high resolution Herschel/PACS $70 \mu \mathrm{m}$ images, we find an even larger $\mathcal{C}_{70}$ for a smaller aperture size $(\sim 200 \mathrm{pc})$ than previous findings by $\mathrm{Li}$ et al. (2010) and Calzetti et al. (2010) with $\sim 700$ pc regions and whole galaxies, respectively, which reveals a diffuse $70 \mu \mathrm{m}$ emission fraction of about $50 \%$ beyond our $200 \mathrm{pc}$ regions. We hypothesize this fraction is due to heating of dust by older stars not associated with current star formation. With the comparison to a simple model, we find this increasing trend of $\mathcal{C}_{70}$ with decreasing region sizes to be physically related to the associated star formation timescale, i.e., smaller regions related to a shorter star formation timescale have less fractional contribution from dust heated by stellar populations that are not related to current star formation.

\subsection{Analysis with 100 and $160 \mu \mathrm{m}$}

To perform the analysis at 100 and $160 \mu \mathrm{m}$, we also process the PACS images of these two wavelengths according to the methodology described in Section $3.3(\mathrm{H} \alpha$ and $\mathrm{Br} \gamma$ images as well), in order to degrade all images to the same resolution of 6."9 for $100 \mu \mathrm{m}$ and $11^{\prime \prime} .5$ for $160 \mu \mathrm{m}$. The adopted aperture radii are $8^{\prime \prime}$ for the images at the $100 \mu \mathrm{m}$ resolution and $12^{\prime \prime}$ for the images at the $160 \mu \mathrm{m}$ resolution, corresponding to physical sizes of about $330 \mathrm{pc}$ and $400 \mathrm{pc}$. With these aperture sizes, 65 and 47 regions for NGC 5055 and 86 and 62 regions for NGC 6946 are selected at the 100 and $160 \mu \mathrm{m}$ resolutions, respectively (Figure 10). The final median uncertainties (and uncertainty ranges) are about $11 \%(10 \%-23 \%)$ at $100 \mu \mathrm{m}$ and $12 \%(10 \%-26 \%)$ at $160 \mu \mathrm{m}$, while the $\mathrm{Br} \gamma$ measurements have similar uncertainties as those mentioned in Section 3.3.2 at $70 \mu \mathrm{m}$ resolution. With the extinction corrected $\mathrm{Br} \gamma$ at matched resolution as the reference SFR, the correlations between both 100 and $160 \mu \mathrm{m}$ and SFR are shown in Figure 11. The fit results and derived Calibration Coefficients (where applicable) are listed in Table 6.

The data for NGC 6946 show a correlation with slope close to unity between the longer wavelengths of FIR bands and the SFR, and the corresponding $\mathcal{C}_{100}$ and $\mathcal{C}_{160}$ values are derived. However, the best-fit slopes for the NGC 5055 data deviate significantly from unity, being smaller than 1 . Moreover, the slope for the $160 \mu \mathrm{m}$ resolution data flattens dramatically. To understand the physical reason behind this flattening, we use the $70 \mu \mathrm{m}$ emission as a gauge to study the behavior of longer wavelengths at different IR brightness (Figure 12). For this analysis, we match the $70 \mu \mathrm{m}$ image of the two galaxies to the $100 \mu \mathrm{m}$ resolution and to the $160 \mu \mathrm{m}$ resolution, respectively, by performing the same convolution process described above, where each image is convolved with the PSF of the other image. Thus the resolutions of the matched images are about 8.7 at $100 \mu \mathrm{m}$ and 12 ."7 at $160 \mu \mathrm{m}$, and the adopted aperture radii are $10^{\prime \prime}$ and $14^{\prime \prime}$, respectively. The inner and outer annuli radii are again two times and four times the aperture radii. The ratios of $\Sigma(100) / \Sigma(70)$ and $\Sigma(160) / \Sigma(70)$ are plotted as a function of $\Sigma(70)$ for both galaxies, 


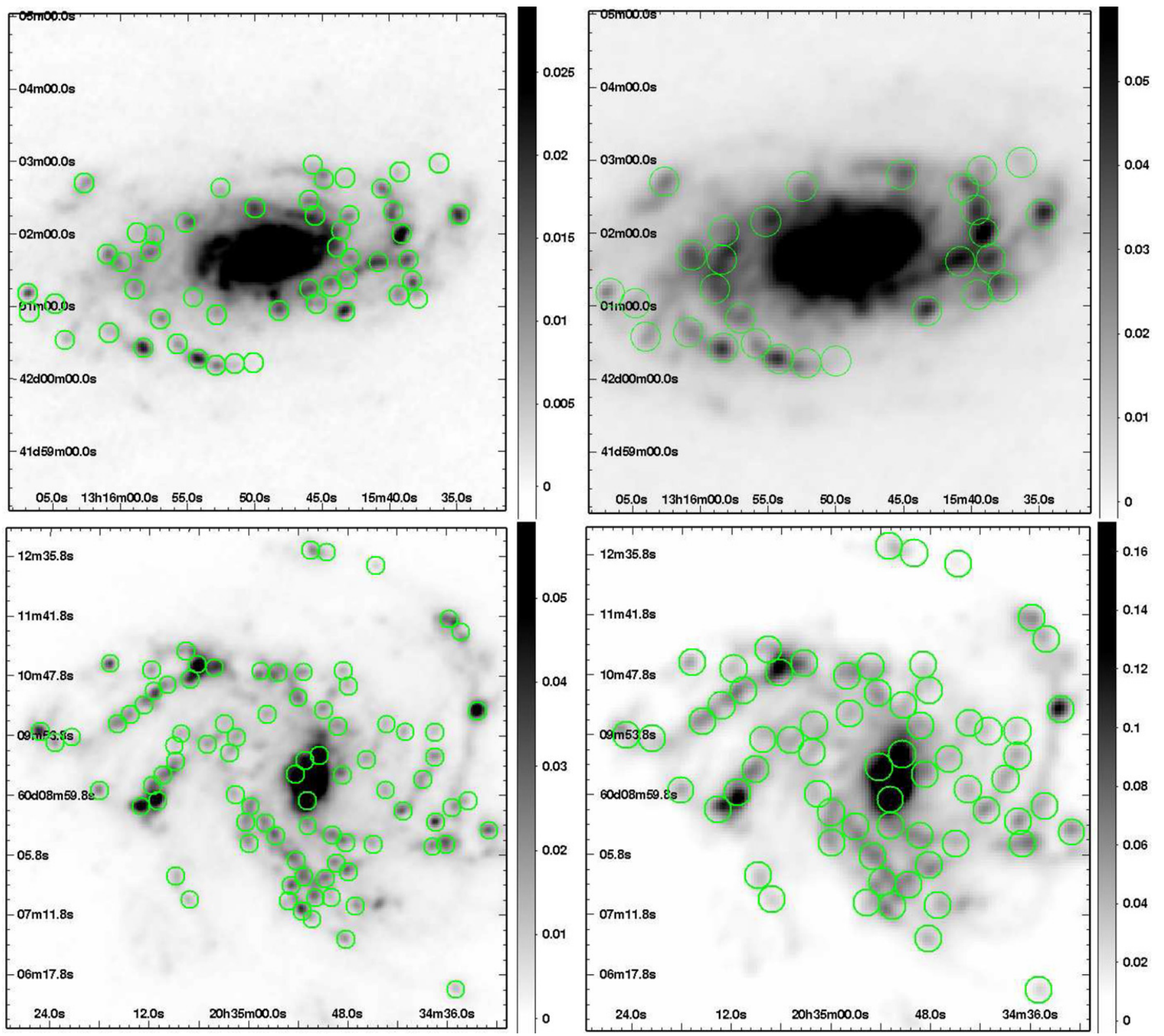

Figure 10. From top left to bottom right: $100 \mu \mathrm{m}$ and $160 \mu \mathrm{m}$ images for NGC 5055 and $100 \mu \mathrm{m}$ and $160 \mu \mathrm{m}$ images for NGC 6946. The green circles are the apertures we adopted for $100 \mu \mathrm{m}$ and $160 \mu \mathrm{m}$ resolution analysis, respectively. North is up and east is left. The image size is about $410^{\prime \prime} \times 410^{\prime \prime}$ (about $15.8 \times 15.8 \mathrm{kpc}$ ) for each panel of NGC 5055 and about $450^{\prime \prime} \times 450^{\prime \prime}$ (about $14.8 \times 14.8 \mathrm{kpc}$ ) for each panel of NGC 6946. The convolved Br $\gamma$ images are not shown but the uneven background is worse with lower resolution. The units for the grayscale bars are all Jy per pixel, while the $100 \mu \mathrm{m}$ images have pixel sizes of 1 .'7 and the $160 \mu \mathrm{m}$ images have pixel sizes of 2". 85 .

(A color version of this figure is available in the online journal.)

and a linear fit through the data in $\log -\log$ space is drawn on each panel. For the $\Sigma(160) / \Sigma(70)$ ratio, we use the model mentioned in Section 4.3 (adding the $160 \mu$ m output) to produce a model prediction, shown as dashed lines on the lower panels. The difference of $\Sigma(160) / \Sigma(70)$ ratios between NGC 5055 and NGC 6946 is obvious, and the model prediction agrees with the NGC 6946 data. Since any resolution complication is taken out by matching the resolution among images, the steepening trend of ratios for NGC 5055 is most likely coming from the fact that this galaxy is more inclined than NGC 6946 which increases line-of-sight overlap and confusion and/or possible over-subtraction of the star forming regions contributing to the local 70 micron emission. This is further supported by the exacerbation of the effect for increasing wavelength (lower resolution), which increases the line-of-sight confusion. In addition, NGC 5055 has higher $\Sigma(100) / \Sigma(70)$ and $\Sigma(160) / \Sigma(70)$ ratios on average than NGC 6946, which agrees with the result that NGC 5055 has globally colder dust as indicated by Draine et al. (2007) and Dale et al. (2012), and as discussed in Section 4.2. Thus, the reason for a flat trend of $\Sigma(100) / \Sigma(70)$ and $\Sigma(160) / \Sigma(70)$ ratios versus $\Sigma(70)$ for NGC 5055 , which leads to a flat trend of the 100 and $160 \mu \mathrm{m}$ correlation with the SFR, is summarized as: (1) a combination of decreasing resolution and high inclination, which produces higher line-of-sight overlap, and results in a higher degree of uncertainty when removing the diffuse contribution of NGC 5055 using local background subtraction, and/or causes over-subtraction of the star forming regions contributing to the local 70 micron emission, and/or 


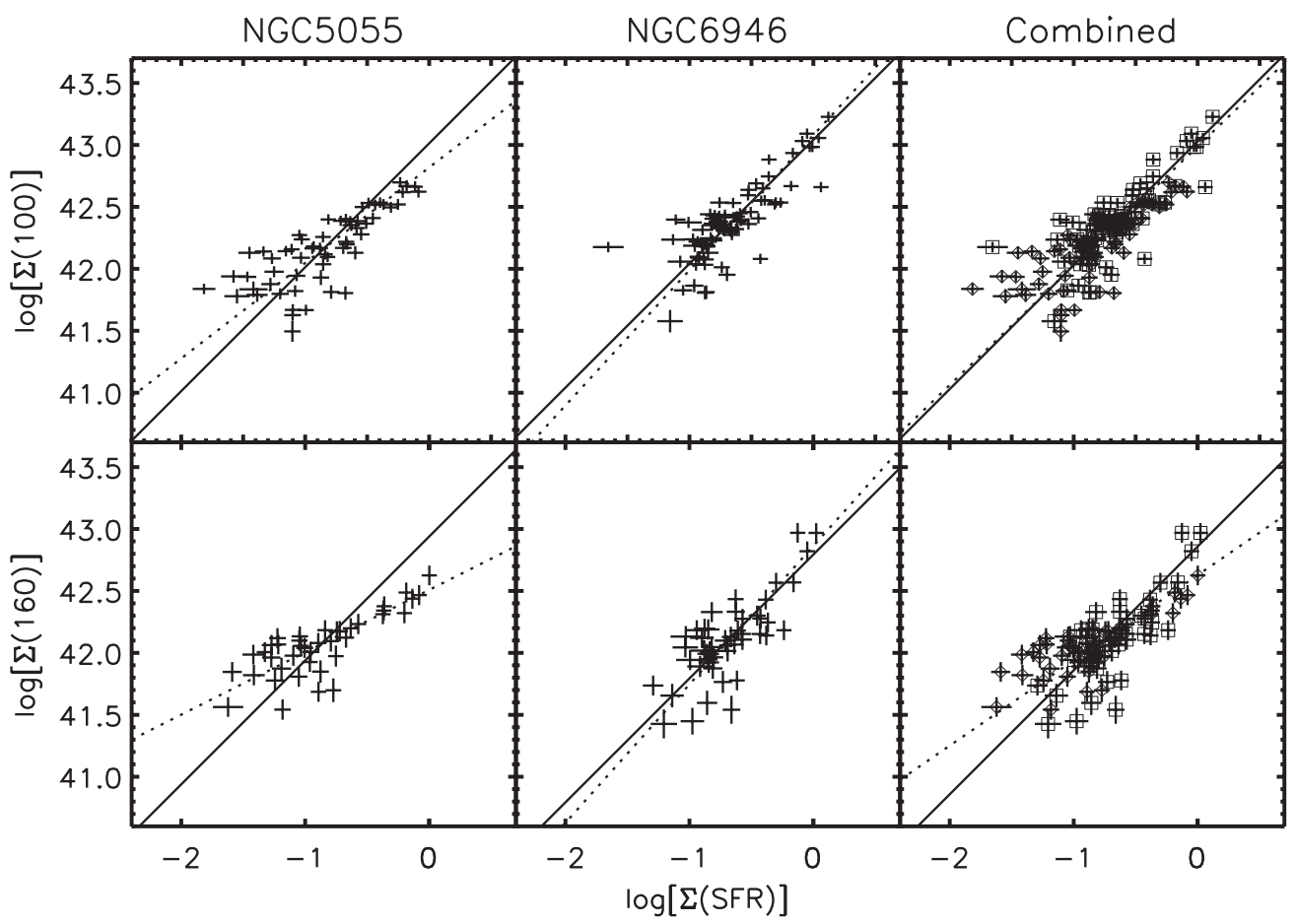

Figure 11. $\Sigma(100)$ and $\Sigma(160)$ as a function of $\Sigma(\mathrm{SFR})$, from extinction corrected $\Sigma(\mathrm{Br} \gamma$ ). The vertical panels are data for NGC 5055 (left), NGC 6946 (middle) and both combined (right), respectively. In the right panel, open diamonds are for NGC 5055 and open squares are for NGC 6946. Dotted lines are linear fits in $\log -\log$ space and solid lines are linear fit with unity slope in $\log -\log$ space. We notice the flattening trend of NGC 5055 with longer wavelength, which is mainly due to a combined effect of decreasing resolution and higher inclination for this galaxy than NGC 6946. The units are $M_{\odot} \mathrm{yr}^{-1} \mathrm{kpc}^{-2}$ for SFR surface density and $\mathrm{erg} \mathrm{s}^{-1} \mathrm{kpc}^{-2}$ for LSD separately.

Table 6

Fitting Results: $\log [\Sigma(100)]$ and $\log [\Sigma(160)]$ versus $\log [\Sigma(\mathrm{SFR})]$

\begin{tabular}{lccccc}
\hline \hline \multicolumn{7}{c}{ Fitting Parameters } & & \\
\cline { 2 - 4 }$(1)$ & $a_{0}$ & $b_{0}$ & $a_{1}$ & $\mathcal{C}$ & $\sigma$ \\
& $(2)$ & $(3)$ & $(4)$ & $(5)$ & $(6)$ \\
\hline NGC 5055 & $42.818(0.026)$ & $0.771(0.028)$ & $43.013(0.011)$ & $\ldots$ & 0.20 \\
NGC 6946 & $43.079(0.027)$ & $1.091(0.037)$ & $43.041(0.010)$ & $0.91(0.02)$ & 0.21 \\
Combined & $42.987(0.018)$ & $0.963(0.023)$ & $43.029(0.007)$ & $0.94(0.02)$ & 0.22 \\
\hline \multicolumn{7}{c}{$100 \mu \mathrm{m}$} \\
\hline NGC 5055 & $42.508(0.034)$ & $0.503(0.035)$ & $42.938(0.017)$ & $\ldots$ & 0.15 \\
NGC 6946 & $42.863(0.050)$ & $1.117(0.068)$ & $42.793(0.017)$ & $1.61(0.06)$ & 0.22 \\
Combined & $42.628(0.026)$ & $0.688(0.030)$ & $42.859(0.012)$ & $\ldots$ & 0.20 \\
& & & & & \\
\hline
\end{tabular}

Notes. Column 1: data set; Columns 2-4: fit parameters as in $y=a_{0}+b_{0} x$ and $y=a_{1}+x$; Column 5: derived Calibration Coefficient, $\mathcal{C}=10^{43-a_{1}} ;$ Column 6 : $1 \sigma$ dispersion of the data around the linear fit.

(2) the regions in NGC 5055 have colder dust than that in NGC 6946, possibly a consequence of self-shielding by dust in the H II regions of NGC 5055, which have high extinction, combined with a globally lower luminosity for the interstellar radiation field (Section 4.2), and contribute more to the longer wavelengths.

Although we derive $\mathcal{C}_{100}$ and $\mathcal{C}_{160}$ from the NGC 6946 data, which has reasonably linear behavior, the difference between NGC 5055 and NGC 6946 questions the applicability of our Calibration Coefficient to general cases for 100 and $160 \mu \mathrm{m}$. Conservatively, one might assume that the longer wavelengths are not as good SFR indicators as the shorter wavelength $70 \mu \mathrm{m}$ emission, but we will revisit this issue with a larger sample of galaxies with $\mathrm{Br} \gamma$ imaging.

\section{SUMMARY}

We use archival CFHT/WIRCam $\mathrm{Br} \gamma$ narrowband and $K s$ broadband observations for two galaxies, NGC 5055 and NGC 6946, to produce $\mathrm{Br} \gamma$ emission line maps. We adopt the $\mathrm{Br} \gamma$ emission as our reference SFR indicator for calibrating SFR(70) with the Herschel/PACS $70 \mu \mathrm{m}$ data from the KINGFISH observations. The H II region luminosity functions of the two galaxies have a slope consistent with -1 , which agrees with previous studies using $\mathrm{H} \alpha$, and lends credence to the fact that the $\mathrm{Br} \gamma$ map processing, continuum subtraction and absolute photometric calibration are reliable. A comparison with the free-free emission shows the same consistency in the $\mathrm{Br} \gamma$ maps.

The SINGS and LVL $\mathrm{H} \alpha$ images are used for extinction correction, assuming Case $\mathrm{B}$ recombination. Both the $\mathrm{Br} \gamma$ and $\mathrm{H} \alpha$ images are convolved with the observed Herschel PSF of the $70 \mu \mathrm{m}$ band and the $70 \mu \mathrm{m}$ band is also convolved with the $\mathrm{Br} \gamma$ PSF, to better match the resolution. Sources are selected based on presence of both IR and $\mathrm{Br} \gamma$ emission peaks; thus only the $\mathrm{H}$ II regions that are bright enough (to have detected $\mathrm{Br} \gamma$ emission) with enough dust (to have IR emission) are selected for our calibrations of SFR(70) and analysis. Aperture photometry is performed on the sources using the standard approach of employing annuli around the photometry apertures to remove the local background.

We use all sources with $\mathrm{S} / \mathrm{N}>3$ at a depth of $L(\mathrm{Br} \gamma) \sim 10^{36.6}$ which corresponds to star clusters with mass $\sim 3-6 \times 10^{4} M_{\odot}$, assuming an average age of 4-5 Myr and a Kroupa (2001) stellar IMF. With the estimates of Cerviño et al. (2002) adapted 


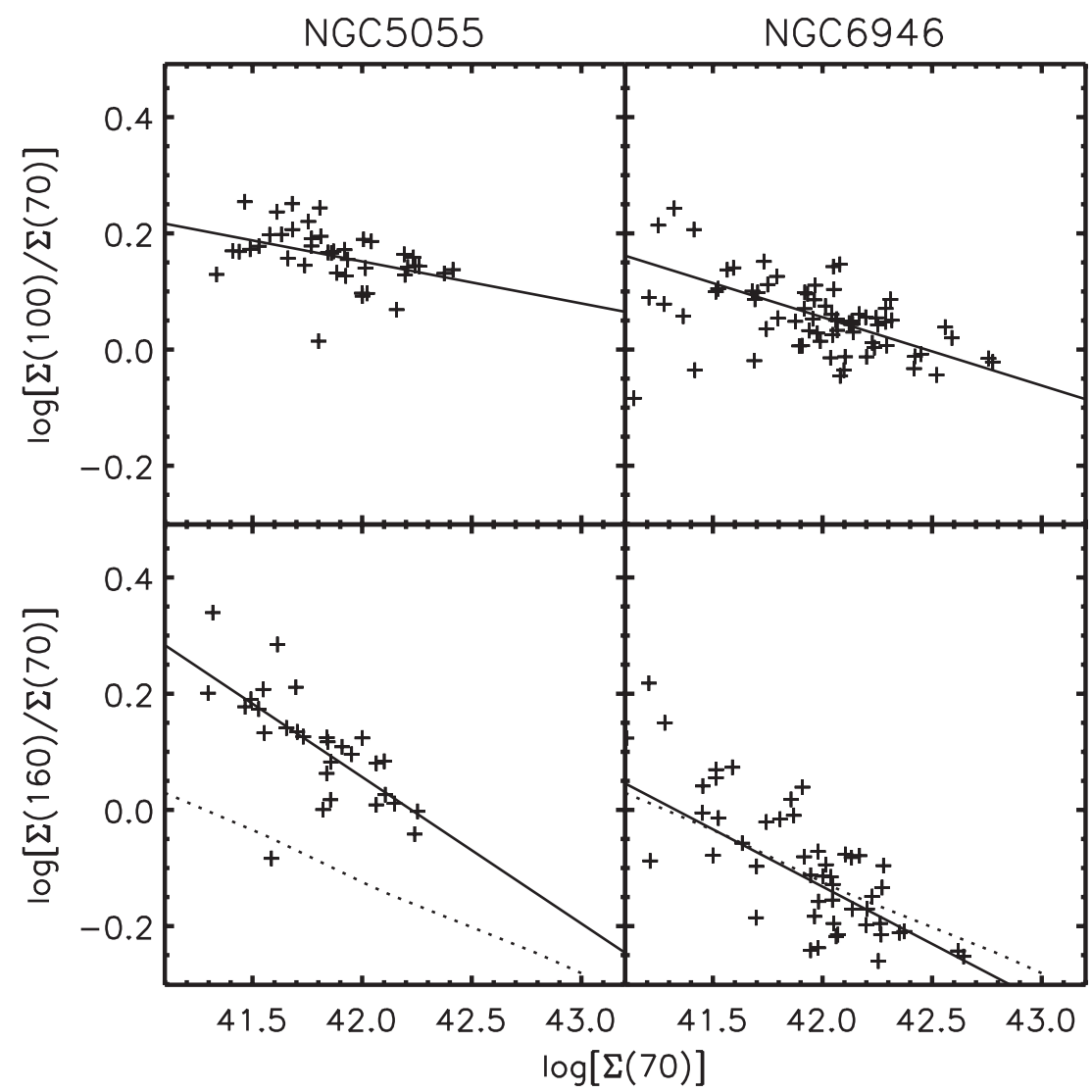

Figure 12. The ratios of $\Sigma(100)$ and $\Sigma(160)$ to $\Sigma(70)$ as a function of $\Sigma(70)$. The solid lines are linear fits through the data in log-log space. Error bars are shown for each data point. The ratios for NGC 5055 are on average higher than the ratios for NGC 6946. The dashed lines on the lower panels are the same expectation from the Draine \& Li (2007) model, which agrees with the NGC 6946 data. The NGC 5055 ratio of $160 \mu \mathrm{m}$ to $70 \mu \mathrm{m}$ is much steeper than the model prediction, and is most likely related to the higher inclination of NGC 5055, since the resolutions are matched between $70 \mu \mathrm{m}$ and $160 \mu \mathrm{m}$ for this analysis. The corresponding $U$, the average stellar radiation field strength defined by Draine \& $\mathrm{Li}(2007)$ and adopted in the model, are about 0.94 to 3.07 and 1.50 to 4.97 for the range of $\Sigma(160) / \Sigma(70)$ ratio for NGC 5055 and NGC 6946, respectively. The unit is $\mathrm{erg} \mathrm{s}^{-1} \mathrm{kpc}^{-2}$ for LSD.

to our IMF, it gives a fluctuation in the ionizing photon flux of less than $30 \%$. Thus random fluctuation in the ionizing photon flux is not a concern. Our analysis finds the following.

1. The $70 \mu \mathrm{m}$ emission can be combined with the $\mathrm{H} \alpha$ emission to produce an unbiased SFR indicator that combines dust obscured and unobscured star formation. The two LSDs are combined as $\Sigma_{\mathrm{H} \alpha, \text { obs }}+(0.011 \pm 0.001) \Sigma(70)$, where the proportionality constant between the observed $\mathrm{H} \alpha$ and $70 \mu \mathrm{m}$ emission is derived for NGC 6946 (Table 4). The same constant for NGC 5055 would be higher, but this galaxy suffers from strong inclination effects (Section 4).

2. Correlations between the $\Sigma(70)$ and $\Sigma($ SFR $)$ give the Calibration Coefficient estimate, $\mathcal{C}_{70,200 \mathrm{pc}}=1.18( \pm 0.02)$ (Table 5), after combining data from both galaxies. Comparison between the derived $\mathcal{C}_{70}$ in this work and those from Calzetti et al. (2010) and Li et al. (2010) reveals a trend of increasing $\mathcal{C}_{70}$ with decreasing region size (Figure 9), implying that in galaxies about $50 \%$ of the $70 \mu \mathrm{m}$ emission outside of a $200 \mathrm{pc}$ scale centered on bright $\mathrm{H}$ II regions is unrelated to current star formation. The trend is still present even when the local background is not removed from the $70 \mu \mathrm{m}$ photometry. Further analysis with a simple model comparison links this trend to a relation with different star formation timescales, i.e., larger regions have longer star formation timescales and thus have more IR emission produced by dust heated by stellar populations that are not related to current star formation as traced by the ionizing photons. This result, first discussed in $\mathrm{Li}$ et al. (2010), is confirmed here by exploiting a larger range of physical sizes.

Whenever applying a SFR(IR) calibration, it is essential to choose a suitable SFR(IR) at the relevant physical scale (star formation timescale) of the system. As shown by Li et al. (2010, Equation (5) in the paper), a metallicity change from about solar to extreme sub-solar could mean a factor two change in the Calibration Coefficient, which should also be taken into consideration when dealing with low metallicity systems.

A similar analysis for PACS 100 and $160 \mu \mathrm{m}$ is presented in Section 4.4. Although the results are limited due to the smaller number statistics with lower resolution and the dispersions in the data are similar to or slightly larger than that of the $70 \mu \mathrm{m}$, the increased variations between the two galaxies at longer wavelengths hint that they are possibly not as good SFR indicators as $70 \mu \mathrm{m}$ due to more significant contributions from dust heated by stellar populations that are not related to current star formation and its variations in different galaxies. This is in agreement with the conclusions of Calzetti et al. (2010) for whole galaxies and Lawton et al. (2010) for $\mathrm{H}$ II regions in the LMC and SMC. Ultimately, given the differences we find in the calibration constants of the two galaxies analyzed in this study, we will need to investigate a larger sample, in order to confirm the mean values derived in this work. We expect to perform the same analysis using a ten-fold larger sample in the near future. 
This work is based in part on observations made with Herschel, a European Space Agency Cornerstone Mission with significant participation by NASA. Partial support for this work was provided by NASA through the award 1369560 issued by JPL/Caltech. This work has also been partially supported by the NASA-ADAP grant NNX10AD08G. The research of C.D.W. is supported by grants from the Natural Sciences and Engineering Research Council of Canada.

The authors would like to thank Daniel Devost for the WIRCam observations with RUNID 07AD86 for NGC 5055 and 07BD91 for NGC 6946.

This work has made use of the NASA/IPAC Extragalactic Database (NED), which is operated by the Jet Propulsion Laboratory, California Institute of Technology, under contract with the National Aeronautics and Space Administration.

The authors thank an anonymous referee for valuable comments that have helped improve this paper.

\section{REFERENCES}

Alonso-Herrero, A., Rieke, G. H., Rieke, M. J., et al. 2006, ApJ, 650, 835 Boquien, M., Calzetti, D., Kramer, C., et al. 2010, A\&A, 518, L70 Bresolin, F., Garnett, D. R., \& Kennicutt, R. C., Jr. 2004, ApJ, 615, 228 Calzetti, D. 1997, AJ, 113, 162

Calzetti, D. 2012, arXiv:1208.2997

Calzetti, D., Armus, L., Bohlin, R. C., et al. 2000, ApJ, 533, 682

Calzetti, D., Kennicutt, R. C., Engelbracht, C. W., et al. 2007, ApJ, 666, 870

Calzetti, D., Kennicutt, R. C., Jr., Bianchi, L., et al. 2005, ApJ, 633, 871

Calzetti, D., Kinney, A. L., \& Storchi-Bergmann, T. 1996, ApJ, 458, 132

Calzetti, D., Liu, G., \& Koda, J. 2012, ApJ, 752, 98

Calzetti, D., Wu, S.-Y., Hong, S., et al. 2010, ApJ, 714, 1256

Cerviño, M., Valls-Gabaud, D., Luridiana, V., \& Mas-Hesse, J. M. 2002, A\&A, 381,51

Crocker, A. F., Calzetti, D., Thilker, D. A., et al. 2013, ApJ, 762, 79

Dale, D. A., Aniano, G., Engelbracht, C. W., et al. 2012, ApJ, 745, 95

Dale, D. A., Cohen, S. A., Johnson, L. C., et al. 2009, ApJ, 703, 517

Dopita, M. A., Groves, B. A., Sutherland, R. S., \& Kewley, L. J. 2003, ApJ, 583,727

Draine, B. T. 2011, ApJ, 732, 100

Draine, B. T., Dale, D. A., Bendo, G., et al. 2007, ApJ, 663, 866

Draine, B. T., \& Li, A. 2007, ApJ, 657, 810

Elbaz, D., Dickinson, M., Hwang, H. S., et al. 2011, A\&A, 533, A119

Ferguson, A. M. N., Wyse, R. F. G., Gallagher, J. S., III, \& Hunter, D. A. 1996, AJ, 111, 2265

Hao, C.-N., Kennicutt, R. C., Johnson, B. D., et al. 2011, ApJ, 741, 124

Hunt, L. K., \& Hirashita, H. 2009, A\&A, 507, 1327

Jones, L. V., Elston, R. J., \& Hunter, D. A. 2002, AJ, 124, 2548
Kennicutt, R. C., Calzetti, D., Aniano, G., et al. 2011, PASP, 123, 1347

Kennicutt, R. C., Jr. 1998, ARA\&A, 36, 189

Kennicutt, R. C., Jr., Armus, L., Bendo, G., et al. 2003, PASP, 115, 928

Kennicutt, R. C., Jr., Calzetti, D., Walter, F., et al. 2007, ApJ, 671, 333

Kennicutt, R. C., Jr., Edgar, B. K., \& Hodge, P. W. 1989, ApJ, 337, 761

Kennicutt, R. C., Jr., \& Evans, N. J. 2012, ARA\&A, 50, 531

Kennicutt, R. C., Jr., Hao, C.-N., Calzetti, D., et al. 2009, ApJ, 703, 1672

Kennicutt, R. C., Jr., Lee, J. C., Funes, S. J., et al. 2008, ApJS, 178, 247

Kobulnicky, H. A., \& Kewley, L. J. 2004, ApJ, 617, 240

Koda, J., Scoville, N., Sawada, T., et al. 2009, ApJL, 700, L132

Kroupa, P. 2001, MNRAS, 322, 231

Lawton, B., Gordon, K. D., Babler, B., et al. 2010, ApJ, 716, 453

Lee, J. C., Gil de Paz, A., Kennicutt, R. C., Jr., et al. 2011, ApJS, 192, 6

Le Floc'h, E., Papovich, C., Dole, H., et al. 2005, ApJ, 632, 169

Leitherer, C., Schaerer, D., Goldader, J. D., et al. 1999, ApJS, 123, 3

Leroy, A. K., Bigiel, F., de Blok, W. J. G., et al. 2012, AJ, 144, 3

Leroy, A. K., Walter, F., Brinks, E., et al. 2008, AJ, 136, 2782

Li, Y., Calzetti, D., Kennicutt, R. C., et al. 2010, ApJ, 725, 677

Liu, G., Koda, J., Calzetti, D., Fukuhara, M., \& Momose, R. 2011, ApJ, 735, 63

Magnelli, B., Elbaz, D., Chary, R. R., et al. 2009, A\&A, 496, 57

Moustakas, J., Kennicutt, R. C., Jr., Tremonti, C. A., et al. 2010, ApJS, 190, 233

Murphy, E. J., Bremseth, J., Mason, B. S., et al. 2012, ApJ, 761, 97

Murphy, E. J., Chary, R.-R., Dickinson, M., et al. 2011a, ApJ, 732, 126

Murphy, E. J., Condon, J. J., Schinnerer, E., et al. 2011b, ApJ, 737, 67

Murphy, E. J., Helou, G., Condon, J. J., et al. 2010, ApJL, 709, L108

O’Donnell, J. E. 1994, ApJ, 422, 158

Oey, M. S., Meurer, G. R., Yelda, S., et al. 2007, ApJ, 661, 801

Osterbrock, D. E. 1989, Astrophysics of Gaseous Nebulae and Active Galactic Nuclei (Mill Valley, CA: University Science Books)

Ott, S. 2010, in ASP Conf. Ser. 434, Astronomical Data Analysis Software and Systems XIX, ed. Y. Mizumoto, K.-I. Morita, \& M. Ohishi (San Francisco, CA: ASP), 139

Pellegrini, E. W., Oey, M. S., Winkler, P. F., et al. 2012, ApJ, 755, 40

Pilyugin, L. S., \& Thuan, T. X. 2005, ApJ, 631, 231

Puget, P., Stadler, E., Doyon, R., et al. 2004, Proc. SPIE, 5492, 978

Rahman, N., Bolatto, A. D., Xue, R., et al. 2012, ApJ, 745, 183

Reddy, N., Dickinson, M., Elbaz, D., et al. 2012, ApJ, 744, 154

Rieke, G. H., Alonso-Herrero, A., Weiner, B. J., et al. 2009, ApJ, 692, 556

Rosenberg, M. J. F., van der Werf, P. P., \& Israel, F. P. 2013, A\&A, 550, A12

Roussel, H. 2012, arXiv:1205.2576

Schlafly, E. F., \& Finkbeiner, D. P. 2011, ApJ, 737, 103

Schlegel, D. J., Finkbeiner, D. P., \& Davis, M. 1998, ApJ, 500, 525

Shaver, P. A., McGee, R. X., Newton, L. M., Danks, A. C., \& Pottasch, S. R. 1983, MNRAS, 204, 53

Skibba, R. A., Engelbracht, C. W., Dale, D., et al. 2011, ApJ, 738, 89

Sotnikova, N. Y., \& Rodionov, S. A. 2003, AstL, 29, 321

Thilker, D. A., Braun, R., \& Walterbos, R. A. M. 2000, AJ, 120, 3070

Thilker, D. A., Walterbos, R. A. M., Braun, R., \& Hoopes, C. G. 2002, AJ, 124,3118

Wu, H., Cao, C., Hao, C.-N., et al. 2005, ApJL, 632, L79 